OPEN ACCESS

Edited by:

Felipe Cava,

Umeå University, Sweden

Reviewed by:

Tom Defoirdt,

Ghent University, Belgium

Steven Rutherford,

Genentech, Inc., United States

*Correspondence: E. Fidelma Boyd fboyd@udel.edu

Specialty section:

This article was submitted to Microbial Physiology and Metabolism,

a section of the journal

Frontiers in Microbiology

Received: 18 February 2021 Accepted: 22 September 2021

Published: 25 October 2021

Citation:

Tague JG, Regmi A, Gregory GJ and Boyd EF (2021) Fis Connects Two Sensory Pathways, Quorum Sensing and Surface Sensing, to Control Motility in Vibrio parahaemolyticus. Front. Microbiol. 12:669447. doi: 10.3389/fmicb.2021.669447

\section{Fis Connects Two Sensory Pathways, Quorum Sensing and Surface Sensing, to Control Motility in Vibrio parahaemolyticus}

\author{
Jessica G. Tague, Abish Regmi, Gwendolyn J. Gregory and E. Fidelma Boyd* \\ Department of Biological Sciences, University of Delaware, Newark, DE, United States
}

Factor for inversion stimulation (Fis) is a global regulator that is highly expressed during exponential phase growth and undetectable in stationary phase growth. Quorum sensing (QS) is a global regulatory mechanism that controls gene expression in response to changes in cell density and growth phase. In Vibrio parahaemolyticus, a marine species and a significant human pathogen, the QS regulatory sRNAs, Qrr1 to Qrr5, are expressed during exponential growth and negatively regulate the high cell density QS master regulator OpaR. OpaR is a positive regulator of capsule polysaccharide (CPS) formation, which is required for biofilm formation, and is a repressor of lateral flagella required for swarming motility. In $V$. parahaemolyticus, we show that Fis is a positive regulator of the grr sRNAs expression. In an in-frame fis deletion mutant, grr expression was repressed and opaR expression was induced. The $\Delta$ fis mutant produced CPS and biofilm, but swarming motility was abolished. Also, the fis deletion mutant was more sensitive to polymyxin B. Swarming motility requires expression of both the surface sensing scr $A B C$ operon and lateral flagella laf operon. Our data showed that in the $\triangle$ fis mutant both laf and scrABC genes were repressed. Fis controlled swarming motility indirectly through the QS pathway and directly through the surface sensing pathway. To determine the effects of Fis on cellular metabolism, we performed in vitro growth competition assays, and found that $\Delta$ fis was outcompeted by wild type in minimal media supplemented with intestinal mucus as a sole nutrient source. The data showed that Fis positively modulated mucus components L-arabinose, D-gluconate and $\mathrm{N}$-acetylD-glucosamine catabolism gene expression. In an in vivo colonization competition assay, $\Delta$ fis was outcompeted by wild type, indicating Fis is required for fitness. Overall, these data demonstrate a global regulatory role for Fis in $V$. parahaemolyticus that includes QS, motility, and metabolism.

Keywords: Fis, quorum sensing, motility, metabolism, swarming 


\section{INTRODUCTION}

The factor for inversion stimulation (Fis) is a nucleoid associated protein (NAP) that has two major functions in bacteria, chromosome organization and gene regulation (Azam et al., 1999; Ishihama, 2010). Fis, along with other NAPs, is an important regulator of ribosome, tRNA and rRNA expression (Bokal et al., 1997; Auner et al., 2003; Schneider et al., 2003; Dennis et al., 2004). As a transcriptional regulator, it can act as both an activator and repressor of a large number of genes (Keane and Dorman, 2003; Kelly et al., 2004; Browning et al., 2005; Grainger et al., 2006). As an activator, Fis can directly bind to RNA polymerase to affect transcription, or indirectly control transcription via DNA supercoiling at promoters (Bokal et al., 1997; McLeod et al., 2002; Auner et al., 2003; Cróinín et al., 2006). Fis controls DNA topology by regulating DNA gyrases (gyrA and $g y r B$ ) and DNA topoisomerase I (topA), required for DNA negative supercoiling in Escherichia coli and Salmonella enterica (Schneider et al., 1999; Keane and Dorman, 2003; Weinstein-Fischer and Altuvia, 2007). In enteric species, Fis was shown to be a global regulator that responded to growth phases and abiotic stresses (González-Gil et al., 1996; Goldberg et al., 2001; Kelly et al., 2004; Browning et al., 2005; Bradley et al., 2007; Lautier and Nasser, 2007; Steen et al., 2010; Zhang et al., 2012; Wang et al., 2013; Lv et al., 2018). In E. coli, Fis is one of the most abundant proteins, highly expressed in early exponential phase cells and absent in stationary phase cells, under aerobic growth conditions (Osuna et al., 1995; Mallik et al., 2004). Fis binds to specific sites, however with limited sequence conservation (Finkel and Johnson, 1992). Fis is known as a nucleoid structuring protein that causes DNA bending, allowing for changes in gene expression (Finkel and Johnson, 1992; Bétermier et al., 1994; Skoko et al., 2006). Genome wide studies have shown over a 1,000 binding peaks for Fis in $E$. coli controlling a fifth of chromosomal genes (Grainger et al., 2006; Cho et al., 2008; Kahramanoglou et al., 2011). It has also been shown that many regulatory regions contain multiple and sometimes overlapping Fis binding sites (BS; Hengen et al., 2003; Shao et al., 2008). Genetic, biochemical and structural analysis in E. coli of Fis high affinity binding sites has demonstrated the presence of a 15-bp core sequence flanked by $\mathrm{G} / \mathrm{C}$ (position -7) and $\mathrm{C} / \mathrm{G}$ (position +7 ) basepairs with a central $\mathrm{A} / \mathrm{T}$ rich region (position 0) (Cho et al., 2008; Shao et al., 2008; Kahramanoglou et al., 2011; Hancock et al., 2016).

In E. coli and S. enterica, studies have shown that Fis can control virulence, motility, and metabolism (González-Gil et al., 1996; Kelly et al., 2004; Grainger et al., 2006; Bradley et al., 2007). These studies identified $100 \mathrm{~s}$ of genes whose expression in vivo is either enhanced or repressed by Fis. In S. enterica, the polar flagellum genes, and genes within several pathogenicity islands, were differentially expressed between a $\Delta$ fis mutant and wild type (Osuna et al., 1995; Cróinín et al., 2006; Wang et al., 2013). In the plant pathogen, Dickeya zeae, a $\Delta$ fis mutant strain showed a total of 490 genes significantly regulated by Fis (Ouafa et al., 2012; Prigent-Combaret et al., 2012; Lv et al., 2018). In Vibrio cholerae, it has been shown that Fis modulates expression of the quorum sensing non-coding regulatory sRNAs (Qrr), qrr1 to qrr4 (Lenz and Bassler, 2007). Quorum sensing (QS) is a term used to describe bacterial communication mediate by chemical signals that allows bacteria to control global gene expression in response to cell density changes (Nealson et al., 1970; Fuqua et al., 1994; Gray et al., 1994; Bassler et al., 1997; Bassler, 1999; Miller and Bassler, 2001; Swift et al., 2001; Miller et al., 2002). In $V$. cholerae, it was proposed that Fis acts along with the QS response regulator LuxO, a sigma factor-54 activator, to transcribe the four qrr1 to qrr4 sRNAs. The Qrr sRNAs repressed the master QS high cell density (HCD) regulator HapR and activated the QS low cell density (LCD) regulator AphA. In a $\Delta$ fis mutant in this species, the qrr sRNAs were repressed and hapR was expressed at wild type levels (Lenz and Bassler, 2007). This is the only other study to examine the role of Fis among Vibrio species.

Vibrio parahaemolyticus is a marine halophile and the leading cause of bacterial seafood-borne gastroenteritis worldwide with increased incidences of infection due to climate change (Nair et al., 2007; Su and Liu, 2007; Froelich and Noble, 2016; Froelich and Daines, 2020). A V. parahaemolyticus infection causes inflammatory diarrhea and its main virulence factors are two type 3 secretions systems and their effector proteins (Makino et al., 2003; O'Boyle and Boyd, 2014; Kodama et al., 2015; De Souza Santos and Orth, 2019; Miller et al., 2019). Unlike $V$. cholerae that only produces a single polar flagellum, $V$. parahaemolyticus produces both a polar flagellum and lateral flagella expressed from the Flh (Fli) and Laf loci, respectively (Belas et al., 1986; McCarter et al., 1988; Stewart and McCarter, 2003). The polar flagellum, required for swimming motility, is produced in cells grown in liquid media and is under the control of sigma factor RpoN ( $\sigma 54)$ and its activator FlaK, and sigma factor FliAP $(\sigma 28)$. The lateral flagella, required for swarming motility on solid surfaces, are under the control of RpoN and its activator LafK, and a second $\sigma 28$ factor FliAL (McCarter and Wright, 1993; Stewart and McCarter, 2003; Jaques and McCarter, 2006; Gode-Potratz et al., 2011; Kernell Burke et al., 2015). Disruption of rpoN abolishes all motility, whereas deletion of either of the two $\sigma 28$ sigma factors, FliAP (FliA) or FliAL, abolishes swimming and swarming, respectively (Stewart and McCarter, 2003; Whitaker et al., 2014). Overall, control of motility in the dual flagellar system of $V$. parahaemolyticus differs significantly from monoflagellar systems of enteric species (Klose and Mekalanos, 1998; McCarter, 1999; Prouty et al., 2001). Previously, it was demonstrated that bacterial motility and metabolism require a functional QS pathway in $V$. parahaemolyticus (Kalburge et al., 2017). In this species, it was shown that deletion of the QS response regulator luxO resulted in repression of the five $q r r$ genes and constitutive expression of $o p a R$ (the hapR homolog). The $\Delta l u x O$ mutant had reduced swimming motility, but swarming motility was abolished, while a $\Delta o p a R$ mutant was hyper-motile and swarming proficient (Kalburge et al., 2017). The QS regulator OpaR is a direct repressor of the laf operon, required for lateral flagellum synthesis and swarming motility (Güvener and McCarter, 2003; Jaques and McCarter, 2006; Gode-Potratz and McCarter, 2011; Kernell Burke et al., 2015). OpaR is an activator of the cps 
operon, required for capsular polysaccharide (CPS) formation, an important component of biofilm (McCarter, 1998; Boles and McCarter, 2002; Kim and McCarter, 2007; Kalburge et al., 2017). Additionally, studies have shown that the $V$. parahaemolyticus surface sensing operon $\operatorname{scr} A B C$ activates swarming motility and represses CPS formation by reducing the intracellular levels of c-di-GMP (Boles and McCarter, 2002; Ferreira et al., 2008; Trimble and McCarter, 2011). A deletion of the $s c r A B C$ operon induces high c-di-GMP levels that repress the laf operon and induce cps gene expression (Boles and McCarter, 2002; Ferreira et al., 2008; Trimble and McCarter, 2011). The surface colonization regulatory (Scr) program in V. parahaemolyticus contains a 100 genes, 70 genes of which are involved in swarming motility and 30 genes involved in biofilm formation that are controlled by intracellular c-di-GMP levels (Boles and McCarter, 2002; Kim and McCarter, 2007; Gode-Potratz and McCarter, 2011; Ferreira et al., 2012). In addition, QS can also modulate c-di-GMP levels to control swarming behavior (Gode-Potratz et al., 2011).

Here, we characterized the role of Fis in V. parahaemolyticus, a marine halophile and gastrointestinal pathogen. This work shows that Fis connects the QS and surface sensing signaling pathways in this species to control swarming motility. We determined the expression pattern of fis across the growth curve and constructed an in-frame $\Delta$ fis deletion mutant to examine its role in $V$. parahaemolyticus physiology. For example, the fis mutant produced more capsule and was more sensitive to the antimicrobial peptide polymyxin B. We demonstrated a role of Fis in the QS pathway, specifically its modulation of the five non-coding regulatory sRNAs, qrr1 to qrr5, as well as the QS HCD master regulator, OpaR. The effects of a fis deletion on swimming and swarming motility were determined and an essential role for Fis in swarming motility was uncovered. DNA binding assays and green fluorescent protein (GFP) reporter assays demonstrated Fis regulation of the qrr sRNA genes, the laf lateral flagellum operon and the surface sensing $s c r A B C$ operon. We performed in vitro growth competition assays and an in vivo colonization assay between the $\Delta$ fis mutant and a lacZ knock-in WT strain, WBWlacZ, to demonstrate a fitness effect when fis is deleted. Further, our data show that Fis positively modulates the expression of L-arabinose, D-gluconate and N-acetyl-D-glucosamine (NAG) metabolism genes. This study demonstrates that Fis integrates the QS and surface sensing pathways to control swarming motility and is important for control of metabolism and in vivo fitness.

\section{MATERIALS AND METHODS}

\section{Bacterial Strains, Media, and Culture Conditions}

All strains and plasmids used in this study are listed in Supplementary Table S1. A streptomycin-resistant clinical isolate V. parahaemolyticus RIMD2210633 was used in this study. Unless stated otherwise, all V. parahaemolyticus strains were grown in lysogeny broth (LB) medium (Fischer Scientific, Pittsburgh, $\mathrm{PA}$ ) containing $3 \% \mathrm{NaCl}(\mathrm{LBS})$ at $37^{\circ} \mathrm{C}$ with aeration or M9 minimal media (Sigma Aldrich, St. Louis, MO) supplemented with $3 \% \mathrm{NaCl}$ (M9S). Antibiotics were added to growth media at the following concentrations: ampicillin (Amp), $100 \mu \mathrm{g} / \mathrm{ml}$, streptomycin (Sm), $200 \mu \mathrm{g} / \mathrm{ml}$, tetracycline (Tet), $\quad 1 \mu \mathrm{g} / \mathrm{ml}$, and chloramphenicol $(\mathrm{Cm}), \quad 12.5 \mu \mathrm{g} / \mathrm{ml}$ when required.

\section{Construction of $\Delta$ fis Mutant in V. parahaemolyticus RIMD2210633}

Splicing by overlap extension (SOE) PCR and an allelic exchange method (Ho et al., 1989) were used to construct an in-frame, non-polar deletion mutant of fis (VP2885) in V.parahaemolyticus RIMD2210633. Briefly, primers were designed using $V$. parahaemolyticus RIMD2210633 genomic DNA as a template. All primers used in this study are listed in Supplementary Table S2. SOE PCR was conducted to obtain an $18 \mathrm{bp}$-truncated version of VP2885 (297-bp). The $\Delta$ fis PCR fragments were cloned into the suicide vector pDS132 (Philippe et al., 2004) and named pDS $\Delta$ fis. pDS $\Delta$ fis was then transformed into E. coli strain $\beta 2155 \lambda$ pir (Dehio and Meyer, 1997), and conjugated into V. parahaemolyticus RIMD2210633. Conjugation was conducted by cross streaking both strains onto LB plates containing $0.3 \mathrm{~mm}$ diaminopimelic acid. The colonies were verified for single crossover via PCR. The colonies that had undergone a single crossover were grown overnight in LBS with no antibiotic added and plated onto LBS containing $10 \%$ sucrose to select for double crossover deletion mutants. The gene deletion was confirmed by PCR and sequencing.

\section{Phenotype Assays}

To observe CPS, heart Infusion media containing 1.5\% agar, $2.5 \mathrm{~mm} \mathrm{CaCl}_{2}$, and $0.25 \%$ Congo red dye was used and plates were incubated at $30^{\circ} \mathrm{C}$, as previously described (Güvener and McCarter, 2003). Biofilm assays were conducted using crystal violet staining. Cultures were grown overnight in LBS and then used to inoculate (1:40 dilution) a 96 well plate, grown static at $37^{\circ} \mathrm{C}$. After $24 \mathrm{~h}$, the wells are washed with PBS, stained with crystal violet for $30 \mathrm{~min}$ and then accessed for biofilm formation. The biofilms are then dissolved in DMSO and $\mathrm{OD}_{595}$ was measured. Swimming assays were conducted in $\mathrm{LB} 2 \% \mathrm{NaCl}$ with $0.6 \%$ agar and swarming assays were conducted in heart infusion (HI) media with $2 \% \mathrm{NaCl}$ and $1.5 \%$ agar (Whitaker et al., 2014). To study swimming behavior, a single colony of the bacterium was stabbed into the center of the plate, and plates were incubated at $37^{\circ} \mathrm{C}$ for $24 \mathrm{~h}$. For the swarming assay, plates were spot inoculated on the surface of the media and grown at $30^{\circ} \mathrm{C}$ for $48 \mathrm{~h}$ (Whitaker et al., 2014). For polymyxin B sensitivity assays, overnight bacterial cultures were diluted (1:50) into LBS and grown for $2 \mathrm{~h}$ and then spun down and suspended in $5 \mathrm{ml}$ LBS. Polymyxin B sulphate (Sigma-Aldrich) was added to the cultures (final concentration of $40 \mu \mathrm{g} / \mathrm{ml}$ ) and incubated at $37^{\circ} \mathrm{C}$ for $1 \mathrm{~h}$. For the zero minute time point, an aliquot was obtained before adding the polymyxin $B$, and then aliquots were taken at $30 \mathrm{~min}$ and $60 \mathrm{~min}$ time points. The aliquots were serially diluted and plated to determine the colony forming units (CFUs) at each 
specific time point. Percent survival was calculated by dividing the CFUs at $30 \mathrm{~min}$ and $60 \mathrm{~min}$ with that of $0 \mathrm{~min}$ and multiplied by 100 . For disk assay, similar growth condition as above were used, with $2 \mathrm{~h}$ growth cultures spread plated onto LBS plates. Polymyxin B disks $(100 \mu \mathrm{g}$ of polymyxin in each disk) were placed on each plate and incubated for $24 \mathrm{~h}$ at $37^{\circ} \mathrm{C}$ before the zone of inhibitions were measured. Three technical replicates and two biological replicates were performed for each strain.

\section{Bioinformatics Analysis to Identify Putative Fis Binding Sites}

The regulatory region of each gene cluster of interest from $V$. parahaemolyticus RIMD210633 was obtained using NCBI nucleotide database. Virtual footprint was used to identify putative Fis binding sites using the E. coli Fis consensus binding sequence (Münch et al., 2003). The 229-bp, 416-bp, 371-bp, 385-bp, 153-bp, 385-bp, and 545bp DNA regions upstream of flhA (VP2235-VP2231), lafB (VPA1550-VPA1557), araB (VPA1674), nagB (VPA0038), gntK (VP0063), and scrABC (VPA1513) respectively, were used as inputs for Fis binding. The regulatory regions of qrr1 (193-bp), qrr2 (338-bp), qrr3 (162-bp), qrr4 (287-bp), and qrr5 (177-bp) were also used as inputs. Default settings were used to obtain putative Fis binding sites. A 130-bp sequence of VPA1424 regulatory region was used as a negative control that contains no Fis binding sites. A 229-bp sequence of $g y r A$ regulatory region was used as a positive control for Fis binding, which was previously shown to contain Fis binding sites and to be directly regulated by Fis (Schneider et al., 1999; Keane and Dorman, 2003).

\section{Fis Protein Purification}

Fis was purified using a method previously described with modifications as necessary (Carpenter et al., 2015; Kalburge et al., 2017). Briefly, Fis was cloned into the pMAL-c5x expression vector in which a $6 \mathrm{X}$ His-tag maltose binding protein (MBP) was fused to fis separated by a tobacco etch virus (TEV) protease cleavage site (Liu et al., 2013). Primer pair FisFWDpMAL and FisREVPMAL (Supplementary Table S2) and $V$. parahaemolyticus RIMD2210633 genomic DNA were used to amplify fis (VP2885). The fis PCR product along with purified pMAL-c5x, were digested with NcoI and BamHI, ligated with T4 ligase, and transformed into DH5 $\alpha$. The vector pMAL-c5xfis was purified, sequenced, and then transformed into E. coli BL21 (DE3). A $10 \mathrm{ml}$ portion of E. coli BL21 pMAL-c5xfis overnight cultures were used to inoculate $1 \mathrm{~L}$ of fresh $\mathrm{LB}$ supplemented with $100 \mu \mathrm{g} / \mathrm{ml}$ ampicillin and $0.2 \%$ glucose and grown at $37^{\circ} \mathrm{C}$ until the $\mathrm{OD}$ reached 0.4 , at which point, the culture was induced by adding $0.5 \mathrm{~mm}$ IPTG. The cells were grown overnight at $18^{\circ} \mathrm{C}$. Cells were pelleted at $2,800 \times g$ and resuspended in $15 \mathrm{ml}$ of column buffer ( $50 \mathrm{~mm}$ sodium phosphate, $200 \mathrm{~mm} \mathrm{NaCl}, \mathrm{pH}$ 7.5) supplemented with $0.5 \mathrm{~mm}$ benzamidine, and $1 \mathrm{~mm}$ phenylmethylsulphonyl fluoride. Bacterial cells were lysed using a microfluidizer, spun down at $25,000 \times g$ for $60 \mathrm{~min}$, and the supernatant was collected. The supernatant was passed through a $20 \mathrm{ml}$ amylose resin (New England BioLabs) and washed with 10 column volumes (CVs) of column buffer. Fis fused with 6X His-MBP was then eluted with three CVs of column buffer supplemented with $20 \mathrm{~mm}$ maltose. Using 6X His-TEV protease (1:10, TEV:protein in $50 \mathrm{~mm}$ sodium phosphate, $200 \mathrm{~mm} \mathrm{NaCl}, 10 \mathrm{~mm}$ imidazole, $5 \mathrm{~mm} \mathrm{BME}, \mathrm{pH} 7.5$ ) the fused protein was cleaved at the TEV cleavage site. The cleaved protein was adjusted to $20 \mathrm{~mm}$ imidazole and run through an immobilized metal affinity chromatography column using HisPur Ni-NTA resin to remove the cleaved 6X His-MBP and the 6X His-TEV protease. Mass spectrometry was performed to confirm Fis protein molecular weight and SDS-PAGE was conducted to determine its purity.

\section{Electrophoretic Mobility Shift Assays}

The regulatory regions of genes of interest were used as probes in electrophoretic mobility shift assays (EMSA). The regulatory regions of all five $q r r$ sRNAs were analyzed for binding of Fis. A 193-bp fragment of Pqrr1, a 338-bp fragment of Pqrr2, a 162-bp fragment of Pqrr3, a 287-bp fragment of Pqrr4, and a 177-bp fragment of Pqrr5 regulatory regions were used as probes. A 130-bp probe of VPA1424 regulatory region was used as a negative control and a 229-bp probe of PgyrA regulatory region was used as a positive control. Analysis of Fis binding to flagellum gene clusters included a 161-bp probe of PflhA (VP2235-VP2231) and a 244-bp probe of PlafB (VPA1550-VPA1557). The 545-bp regulatory region of $s c r A B C$ (VPA1513-VPA1515) operon was divided into three probes, probe 1147-bp, probe 2136-bp, and probe 3139-bp. For analysis of metabolism, 138-bp and 152-bp probes of ParaB (VPA1674, L-ribulokinase, L-arabinose catabolism), 154-bp and 120-bp probes of PnagB (VPA0038, glucosamine-6-phosphate isomerase, D-glucosamine catabolism), and a138-bp probe of PgntK (VP0063, gluconokinase, D-gluconate catabolism) were used in EMSAs. The EMSA probes were PCR amplified using Phusion Hifidelity Polymerase in $50 \mu \mathrm{l}$ reaction mixture using respective primers sets listed in Supplementary Table S2 and $V$. parahaemolyticus RIMD2210633 genomic DNA as template. Various molar ratios of purified Fis were incubated with $30 \mathrm{ng}$ of target DNA in binding buffer $(10 \mathrm{~mm}$ Tris, $150 \mathrm{~mm} \mathrm{KCL}, 0.1 \mathrm{~mm}$ dithiothreitol, $0.1 \mathrm{~mm}$ EDTA, 5\% PEG, $\mathrm{pH}$ 7.4) for $20 \mathrm{~min}$ at room temperature. A native acrylamide $6 \%$ gel was prepared and pre-run for $2 \mathrm{~h}\left(200 \mathrm{~V}\right.$ at $\left.4^{\circ} \mathrm{C}\right)$ with $1 \mathrm{x}$ Tris-acetate-EDTA (TAE) buffer, and then $10 \mu \mathrm{l}$ of the target DNA-protein mixture was loaded into consecutive lanes. The gel was run at $200 \mathrm{~V}$ for $2 \mathrm{~h}$ in $1 \mathrm{X}$ TAE buffer at $4^{\circ} \mathrm{C}$, which was then stained in an ethidium bromide bath $(0.5 \mu \mathrm{g} /$ $\mathrm{ml}$ ) for $20 \mathrm{~min}$ and imaged. To examine the specificity of Fis binding sites, first, we used an approach using SOE PCR to create mutations in a Fis BS within the PnagB probe 2. Using this approach, we mutated 5 -sites which resulted in less Fis binding, but did not abolish binding since, in this region, there were multiple potential Fis BS. Therefore, for the other regions of interest we used a different approach, designing shorter DNA probes for each regulatory region that contained a single Fis BS. A second mutated probe was also designed to contain mutations at key nucleotide positions (Supplementary Table S3). EMSA were performed on wild 
type and mutated probes using the same ratios and run on the same gel.

\section{Transcription Reporter Assays}

Green fluorescent protein reporter assays were conducted in V. parahaemolyticus RIMD2210633 and $\Delta$ fis strains. Reporter plasmids were constructed with the regulatory regions of motility genes, flh $A$ and $l a f B$, and metabolic genes, $\operatorname{ara} B, n a g B$ and $g n t K$, upstream of a promoterless $g f p$ gene, as previously described (Gregory et al., 2019). Briefly, primers were designed to amplify the regulatory region upstream of each gene or gene cluster with primer pairs listed in Supplementary Table S2. Each amplified regulatory region was then ligated with the promoterless parent vector pRU1064 (Karunakaran et al., 2005), which had been linearized prior with SpeI, using NEBuilder High Fidelity (HiFi) DNA Assembly Master Mix (New England Biolabs, Ipswich, MA) via Gibson Assembly Protocol (Gibson, 2011). Overlapping regions for Gibson Assembly are indicated in lower case letters in the primer sequence in Supplementary Table S2. Reporter plasmid $\mathrm{P}_{\mathrm{flhA}}-g f p$ encompasses 269-bp of the regulatory region upstream of $f l h A$. Reporter plasmid $\mathrm{P}_{\text {lafB }}-g f p$ encompasses 456-bp of the regulatory region upstream of lafB. Reporter plasmid $\mathrm{P}_{\mathrm{araB}}-g f p$ encompasses 411 -bp of the regulatory region upstream of $a r a B$. Reporter plasmid $\mathrm{P}_{\text {nagB }}-g f p$ encompasses 434-bp of the regulatory region upstream of $n a g B$. Reporter plasmid $\mathrm{P}_{\text {gntK }}-g f p$ encompasses 193-bp of the regulatory region upstream of $g n t K$. Reporter plasmid $\mathrm{P}_{\text {scrABC}}-g f p$ encompasses 545-bp of the regulatory region upstream of the $s c r A B C$ operon. Additionally, the regulatory region of qrr1 to qrr5 were amplified and cloned into the pRU1064 reporter plasmid. The plasmids were transformed into E. coli $\mathrm{Dh} 5 \alpha$, purified and sequenced. Plasmids were then conjugated into wild type and the $\Delta$ fis mutant for further analysis.

Strains were grown overnight with aeration at $37^{\circ} \mathrm{C}$ in LBS with Tet $(1 \mu \mathrm{g} / \mathrm{ml})$. Cells were then pelleted, washed two times with 1X PBS, and diluted 1:100 in LBS. Strains containing the Pqrr and PopaR reporters were grown to LCD (0.4-0.45 $\mathrm{OD})$, washed two times with $1 \mathrm{X}$ PBS, and resuspended to a final $\mathrm{OD}$ of 1.0 before measuring relative fluorescence. The metabolism gene reporters were grown for $20 \mathrm{~h}$ with antibiotic selection. $\mathrm{P}_{\mathrm{araB}}-g f p$ was grown in LBS supplemented with $10 \mathrm{~mm}$ $\mathrm{D}$-arabinose, $\mathrm{P}_{\text {nagB }}-g f p$ was grown in LBS supplemented with $10 \mathrm{~mm}$ D-glucosamine, $\mathrm{P}_{\text {gntK }}-g f p$ was grown in LBS supplemented with $10 \mathrm{~mm}$ D-gluconate. Cells were pelleted and resuspended in $1 X$ PBS. The pRUPlafB reporter assay was performed using cells grown on heart infusion (HI) plates for $16 \mathrm{~h}$. Colonies were scraped from the plate and resuspended in $1 x P B S$ to a final $\mathrm{OD}_{595}$ 0.5. GFP fluorescence was measured with excitation at 385 and emission at $509 \mathrm{~nm}$ in black, clear-bottom 96-well plates on a Spark microplate reader with Magellan software (Tecan Systems Inc.). Specific fluorescence was calculated for each sample by normalizing relative fluorescence to $\mathrm{OD}_{595}$. At least two biological replicates, in triplicate, were performed for each assay. Statistics were calculated using an unpaired Student's $t$-test.

\section{In vitro Growth Competition Assays}

In vitro growth competition assays were performed by diluting an inoculum 1:50 into LBS broth, and separately in M9S supplemented with mouse intestinal mucus or $10 \mathrm{~mm}$ of individual carbon sources, D-glucose, L-arabinose, L-ribose, D-gluconate, D-glucosamine, and NAG. A $\beta$-galactosidase knock in $V$. parahaemolyticus RIMD2210633 strain, WBWlacZ, which was previously shown to grow similarly to wild type in vitro and in vivo, was used for all the competition assays (Whitaker et al., 2012 , 2014). The culture was incubated at $37^{\circ} \mathrm{C}$ for $24 \mathrm{~h}$, serially diluted and plated on LBS plus streptomycin and 5-bromo-4chloro-3-indolyl-B-D-galactoside (X-gal). The competitive index (CI) was determined using the following equation: $\mathrm{CI}=$ ratio out $(\Delta$ fis/WBWlacZ)/ratio in ( $\Delta$ fis/WBWlacZ). A CI of $<1$ indicates WBWlacZ outcompetes the $\Delta$ fis mutant, a CI of $>1$ indicates that the $\Delta$ fis mutant outcompetes WBWlacZ. The ratio of $\Delta f i s$ to WBWlacZ in the inoculum mixture is termed as "Ratio in" and the ratio of $\Delta$ fis to WBWlacZ colonies recovered from the mouse intestine is referred as "Ratio out."

\section{In vivo Colonization Competition Assays}

All mice experiments were approved by the University of Delaware Institutional Animal Care and Use Committee (Whitaker et al., 2012, 2014). Inoculum for competition assays was prepared using overnight cultures of WBWlacZ and $\Delta$ fis diluted into fresh LBS media and grown for $4 \mathrm{~h}$. Exponential phase cultures were then pelleted by centrifugation at $4,000 \times g$, washed and resuspended in PBS. One $\mathrm{ml}$ of WBWlacZ and one $\mathrm{ml}$ of $\Delta$ fis were prepared, corresponding to $1 \times 10^{10} \mathrm{CFU}$ of each strain, based on the previously determined $\mathrm{OD}$ and $\mathrm{CFU}$ ratio. A $500 \mu \mathrm{l}$ aliquot of $\Delta$ fis was combined with $500 \mu \mathrm{l}$ of the WBWlacZ, yielding a total bacterial concentration of $1 \times 10^{10} \mathrm{CFU} / \mathrm{ml}$. The inoculum was serially diluted and plated on LBS agar plate supplemented with $200 \mu \mathrm{g} / \mathrm{ml}$ streptomycin and $8 \mu \mathrm{g} / \mathrm{ml}$ of X-gal to determine the exact ratio of the inoculum. Male C57BL/6 mice aged 6 to 10 weeks were housed under specific-pathogen-free conditions in standard cages in groups (5 per group) and provided standard mouse feed and water ad libitum. Pretreatment of mice with streptomycin was performed as previously described (Whitaker et al., 2012, 2014; Haines-Menges et al., 2014; Kalburge et al., 2017). Mice were inoculated with $100 \mu \mathrm{l}$ of the bacterial suspension and $24 \mathrm{~h}$ postinfection, mice were sacrificed, and the entire gastrointestinal tract was harvested. Samples were placed in $8 \mathrm{ml}$ of sterile $1 \mathrm{x}$ PBS, mechanically homogenized and serially diluted in 1xPBS. Diluted samples were plated for CFUs on LBS, supplemented with streptomycin and X-gal for a blue (WBWlacZ) versus white $(\Delta f i s)$ screen of colonies after incubation at $37^{\circ} \mathrm{C}$ overnight. The competitive index (CI) was determined as described above.

\section{RESULTS}

\section{fis Expression Is Controlled in a Growth Dependent Manner}

In V. parahaemolyticus RIMD2210633, locus tag VP2885 is annotated as a Fis protein homolog, a 98 amino acid protein 
that shows $100 \%$ protein identity to Fis from $V$. cholerae and $82 \%$ amino acid identity to Fis from E. coli. Fis is an abundant protein in E. coli, highly expressed in exponential phase cells and undetected in stationary phase cells grown under aerobic conditions (Azam et al., 1999; Mallik et al., 2004). In V. parahaemolyticus RIMD2210633, we determined the expression pattern of fis across the growth curve, via RNA isolated from wild type cells grown in LBS at $37^{\circ} \mathrm{C}$ aerobically at various optical densities (ODs). Using quantitative real time PCR analysis, fis showed highest expression levels in exponential cells at Ods $0.15,0.25$, and 0.5 and then rapidly declined at Ods 0.8 and 1.0, as cells entered stationary phase (Supplementary Figure S1). These data show that fis in $V$. parahaemolyticus has a similar expression pattern to fis in E. coli and also what has been demonstrated in $V$. cholerae (Azam et al., 1999; Mallik et al., 2004; Lenz and Bassler, 2007).

\section{Fis Positively Regulates the QS Non-coding qrr sRNAs}

One other study has examined the role of Fis in Vibrio and this work showed that in $V$. cholerae Fis was a positive regulator of the QS non-coding regulatory sRNA genes qrrl to qrr4 (Lenz and Bassler, 2007). The qrr1 to qrr4 genes in $V$. parahaemolyticus were homologous to those present in $V$. cholerae and showed identical genome locations in both species for each $q r r$ gene. To determine the role of Fis in the regulation of qrr1 to qrr5 in V. parahaemolyticus, we identified multiple putative Fis binding sites within the regulatory regions of all five sRNAs (Figure 1). To demonstrate Fis binding, we purified the Fis protein and constructed DNA probes of the regulatory region of each $q r r$ sRNA and performed EMSA with increasing concentrations of purified Fis. Fis binding was shown in a concentration dependent manner in all five PqrrFis EMSAs that resulted in a complete shift of the probe (Figures 1A-E). Smearing in the wells at higher Fis concentrations was noted but was not present in a protein only control lane. Slight smearing in the lane at the highest ratios of DNA:protein, is potentially due to multimerization of Fis protein that can occur, but we cannot rule out minute DNA contamination. The regulatory region of gyrA was used as a Fis binding positive control and showed specific concentration dependent binding with a complete shift in the probe in all five qrr genes (Figure 1F). A DNA fragment without a putative Fis binding site was used as a non-binding control and showed non-specific, weak binding, and no complete shift of the probe at any concentration (Figure 1G). To examine the specificity of binding further, we aligned the putative Fis binding sites in the regulatory regions of the qrr genes, most of which had multiple and sometimes overlapping binding sites. However, we identified a single Fis binding motif in the coding region of qrr3 and mutated this conserved sites to examine Fis binding. In these EMSAs, no banding shift was observed in the mutated probe (Figure 1H).

To further examine the role of Fis in $V$. parahaemolyticus qrr gene expression, an in-frame deletion of fis was constructed by deleting 279-bp of VP2885. We examined growth of the $\Delta$ fis mutant in LBS broth and found that it grew identical to wild type $V$. parahaemolyticus (Supplementary Figure S2A). However, on LBS agar plates, the $\Delta$ fis mutant formed a small colony morphology compared to wild type. This phenotype was rescued by complementation with a functional copy of fis under the control of an IPTG inducible promoter, restoring wild type colony morphology (Supplementary Figures S2B,C). Next, we performed transcriptional GFP reporter assays using the regulatory region of each $q r r$ to determine whether deletion of fis affects expression. Cells were grown to $0.4-0.45$ OD and GFP levels measured using relative fluorescence normalized to $\mathrm{OD}$ (specific fluorescence). In $\Delta f i s$, the overall expression of $\mathrm{P}_{\mathrm{qrr}_{2}-}$ $g f p$ to $\mathrm{P}_{\mathrm{qrr} 4}-g f p$ was significantly downregulated compared to wild type $(p<0.01$ and 0.001 , respectively), indicating that Fis is a positive regulator of $q r r 2$, qrr3, and qrr4 in $V$. parahaemolyticus (Figures 2B-D). We observed a reduction in $\mathrm{P}_{\mathrm{qrr} 1}-g f p$ and $\mathrm{P}_{\mathrm{qrr} 5}-g f p$ expression in the $\Delta f i s$ mutant relative to wild type, but this reduction was less significant, which may not be physiologically relevant (Figures 2A,E). Next, we examined whether opaR expression was changed in the $\Delta$ fis mutant using GFP reporter expression assays under the control of the opaR regulatory region. In these assays, $\mathrm{P}_{\mathrm{opaR}^{-}}$ $g f p$ activity was significantly upregulated $(p<0.01)$ in the $\Delta$ fis mutant compared to wild type (Figure 2F). Overall, the data suggest that Fis plays a role in the QS pathway, but is not an essential component, instead it likely modulates expression of the $q r r$ sRNAs and $o p a R$ in early exponential phase cells. The data also suggest that other factors are required to control qrr expression. For example, studies have shown that, AphA, LuxO, OpaR, and LuxT also significantly control qrr expression in Vibrio species (Rutherford et al., 2011; Zhang et al., 2012; Kalburge et al., 2017; Eickhoff et al., 2021; Simpson et al., 2021).

\section{Fis Controls Multiple Phenotypes in V. parahaemolyticus}

OpaR, the QS master regulator, is a positive regulator of CPS required for biofilm formation. We examined the effects of a fis deletion on this phenotype given the changes in expression of opaR in the $\Delta$ fis mutant. CPS production in V. parahaemolyticus manifests as an opaque rough wrinkly colony morphology also known as rugose morphology that is also an indirect measure of c-di-GMP levels that oppositely control CPS and swarming motility (McCarter, 1998; Boles and McCarter, 2002; Trimble and McCarter, 2011; Ferreira et al., 2012; Jones and Wozniak, 2017). In the wild-type strain on Congo red plates, cells formed large opaque wrinkly raised colonies, whereas a $\triangle o p a R$ mutant formed a large smooth colony morphology, indicating CPS is lacking (Figure 3A). The $\Delta$ fis mutant produced a much smaller highly wrinkly colony morphology (Figure 3A). This colony morphology is indicative of overproduction of CPS in bacteria (Jones and Wozniak, 2017).

To investigate whether Fis has any role in antimicrobial peptide resistance, the $\Delta$ fis strain was examined for polymyxin 
A

Pqrr1

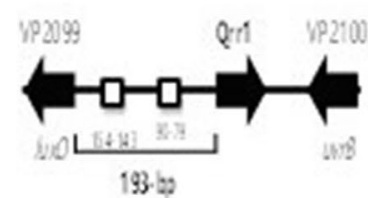

[Fis]
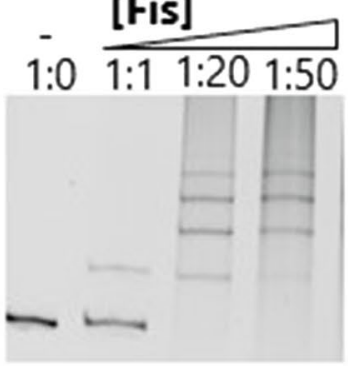

Pqrr5

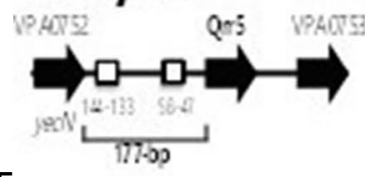

E

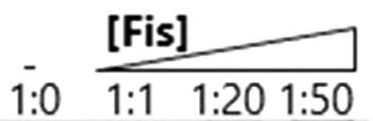

B qrr2

C Pqrr3
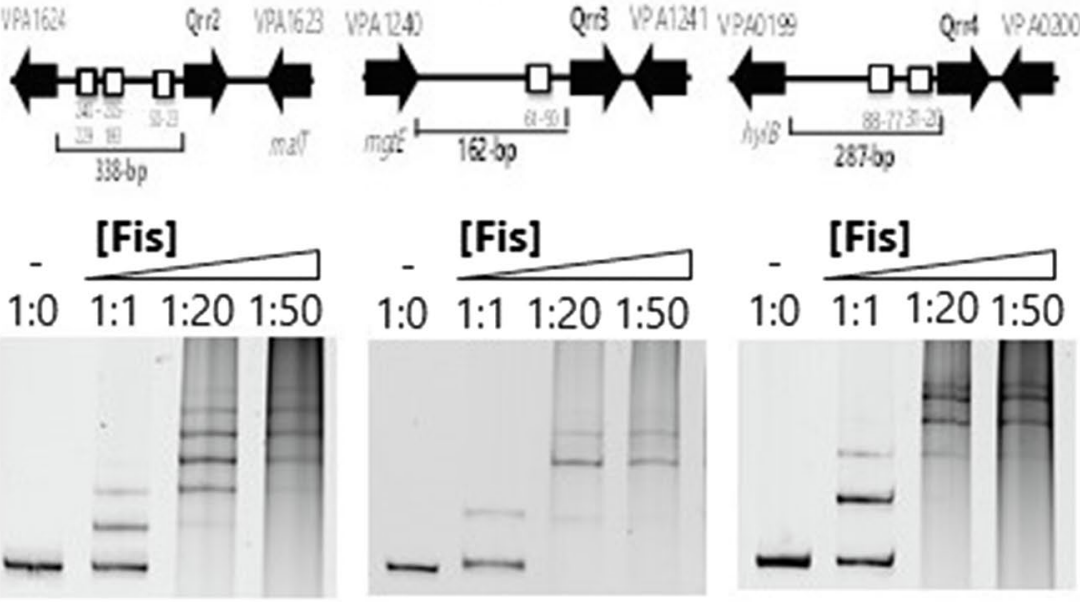

\section{Negative Fis BS control}

$\mathbf{F}$

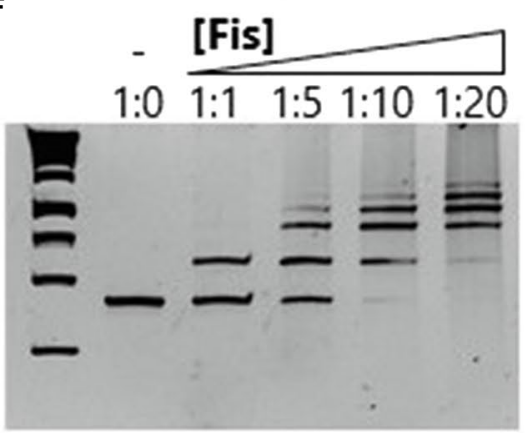

G

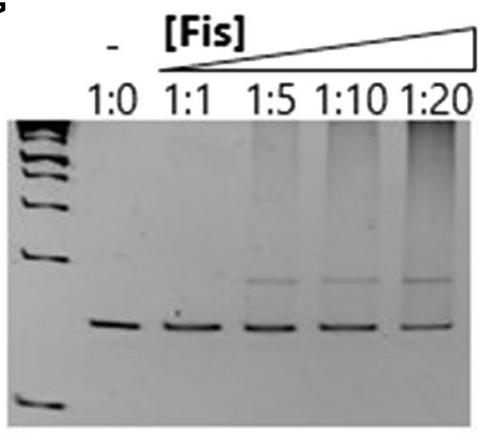

H

\section{Mutated Fis BS probe}

[Fis]

[Fis]

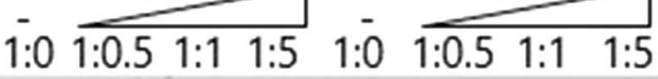

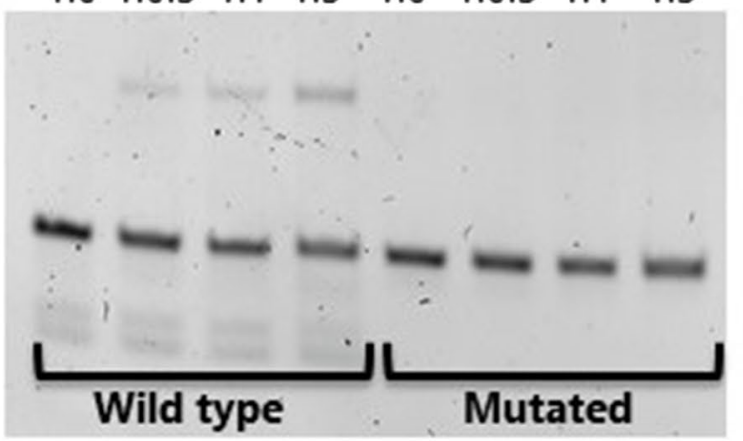

FIGURE 1 | Factor for inversion stimulation binds to the regulatory regions of $V$. parahaemolyticus regulatory sRNAs (A) grr1, (B) grr2, (C) qrr3, (D) qrr4, and (E) qrr5. A putative Fis binding site (BS) is shown as a box in the regulatory region of qrr1 to qrr5. Electrophoretic mobility shift assays (EMSA) using purified Fis and the regulatory regions of grr1 to qrr5. DNA:protein ratios are as follows: 1:0, 1:1, 1:20, 1:50. (F) Positive Fis BS control in gyrA regulatory region showing complete shift at all concentrations. (G) Fis non-binding negative control using DNA with no putative Fis BS showing weak non-specific binding and no shift at lowest concentration. (H) qrr3 probe containing a single Fis BS (wild type) and a mutated BS demonstrating specificity of Fis binding. 


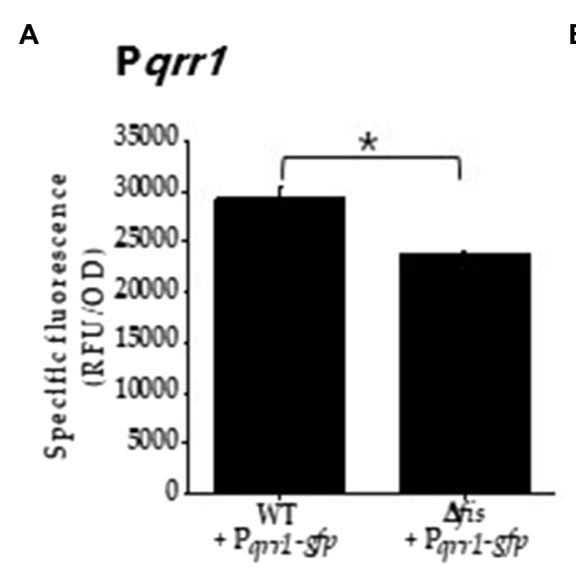

D

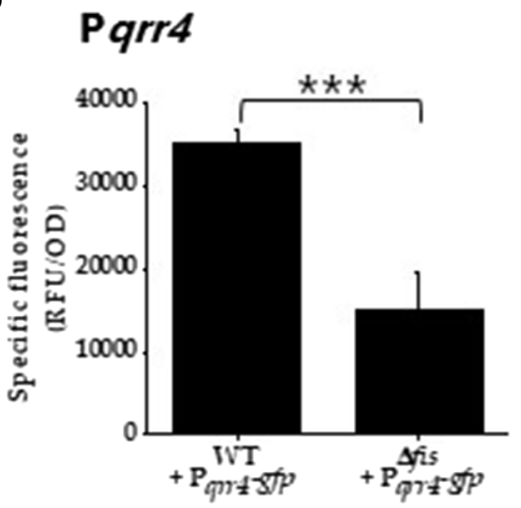

B

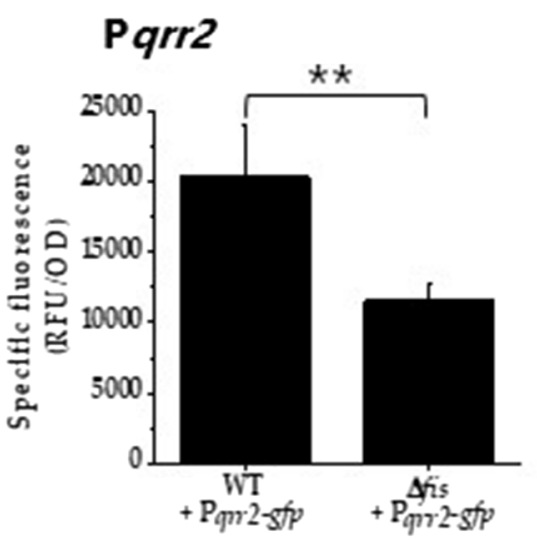

E

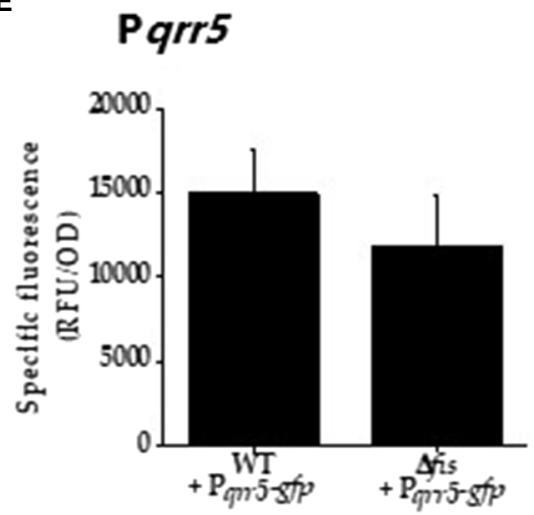

C

\section{Pqrr3}

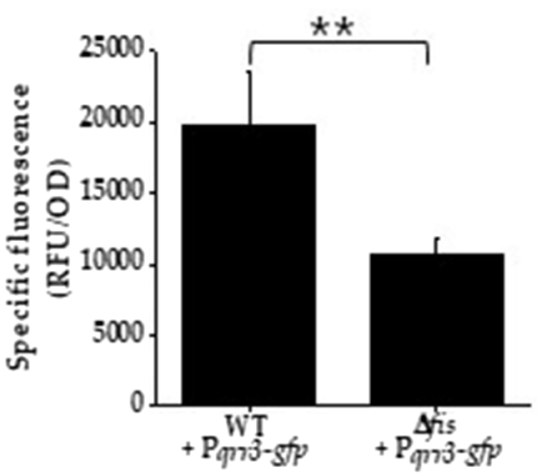

$\mathbf{F}$

\section{PopaR}

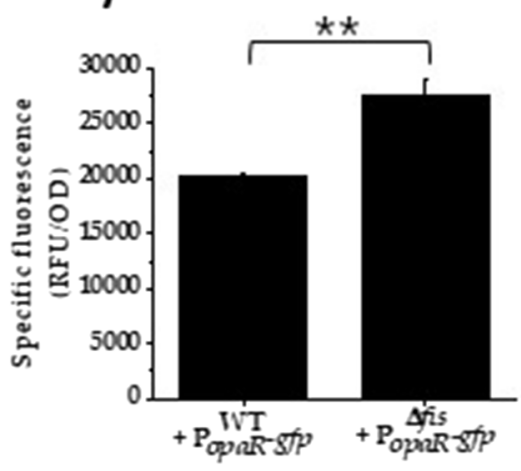

FIGURE 2 | Fis is a positive regulator of the qrr genes. (A) Pqrr1, (B) Pqrr2, (C) Pqrr3, (D) Pqrr4, (E) Pqrr5, and (F) PopaR green fluorescent protein (GFP) transcriptional reporter assays in wild type and the $\Delta$ fis mutant in cultures grown to OD 0.4-0.45 and measured for specific fluorescence (RFU/OD). Means and standard deviations of three biological replicates are plotted. Statistics calculated using a Student's $t$-test $\left({ }^{\star} p<0.05,{ }^{\star *} p<0.01,{ }^{* \star *} p<0.001\right)$.

B sensitivity using disk diffusion and survival assays. Previous work in Vibrio has shown that changes in the bacterial cell wall can result in increased sensitivity to polymyxin B (Haines-Menges et al., 2014; Lubin et al., 2015; McDonald et al., 2018). We used $\Delta r p o E$ as a control, which lacks the sigma factor RpoE that is required for the cell envelope stress response and was previously shown to be polymyxin B sensitive (Haines-Menges et al., 2014). For the disk diffusion assay, the $\Delta$ fis and $\Delta r p o E$ mutants had significantly larger zones of inhibition compared to wild type indicating these mutants are more sensitive to polymyxin B (Figure 4A). To verify this further, we conducted survival assay using $200 \mu \mathrm{g}$ of total polymyxin B in $5 \mathrm{ml}$ of LBS media. CFUs were counted at 0,30 and $60 \mathrm{~min}$ post exposure. Significantly lower CFUs for the $\Delta$ fis were recovered at 30 and $60 \mathrm{~min}$ compared to wild type (Figure 4B). The data demonstrated that Fis is required for polymyxin $\mathrm{B}$ resistance in $V$. parahaemolyticus, although the mechanism remains unknown.

Next, we examined whether deletion of fis affected motility in V. parahaemolyticus, a species that produces both polar and lateral flagella, and where OpaR is a negative regulator of the laf operon (Jaques and McCarter, 2006). Swimming assays demonstrated that the $\Delta$ fis mutant had a defect in motility compared to wild type with reduced spreading across the agar plate (Figure 3B). An $\Delta r p o N$ mutant was also examined as a negative control, and this mutant, as expected, showed no motility (Figure 3B). In swarming assays, the wild-type strain produced a typical swarming cauliflower colony morphology, whereas in the $\Delta$ fis deletion mutant, swarming motility was abolished suggesting lateral flagella are absent (Figure 3C). Complementation of the $\Delta$ fis mutant with a functional copy of the fis gene under the control of an IPTG-inducible promoter rescued swarming and swimming motility (Supplementary Figure S3).

\section{Fis Is Necessary for Motility in V. parahaemolyticus}

To further investigate Fis control of swimming and swarming motility, using bioinformatics, we identified multiple putative Fis binding sites within the regulatory regions of both the polar and lateral flagella biosynthesis operons (Figures 5A,E). We performed EMSA analysis using purified Fis and DNA probes of the regulatory regions of the polar flagellum operon (flh loci VP2235-PV2231) and the lateral flagellum operon (laf 
A

\section{Colony morphology and CPS production}
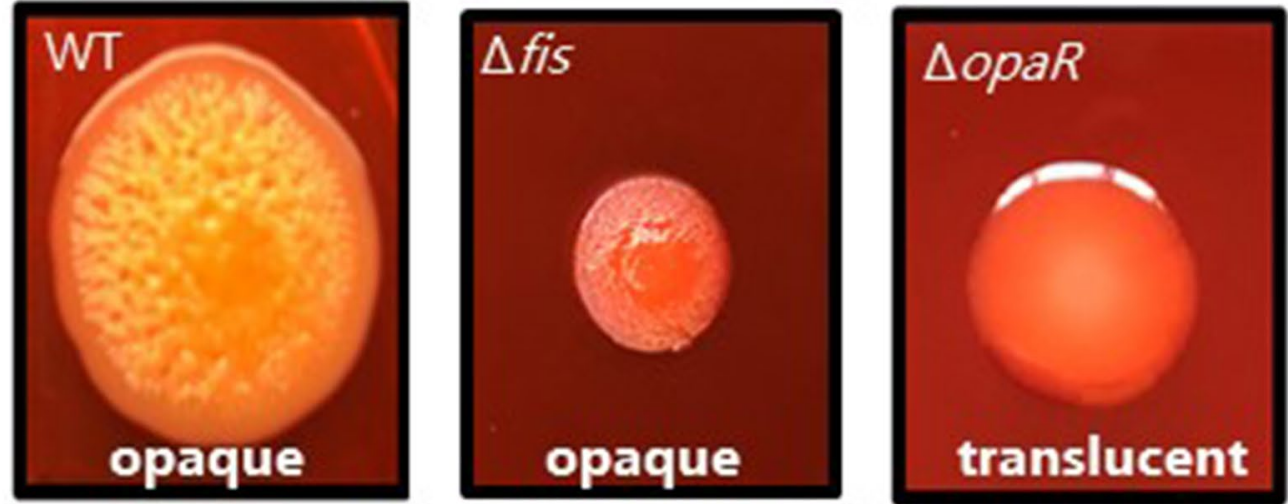

B

\section{Swimming motility assay}
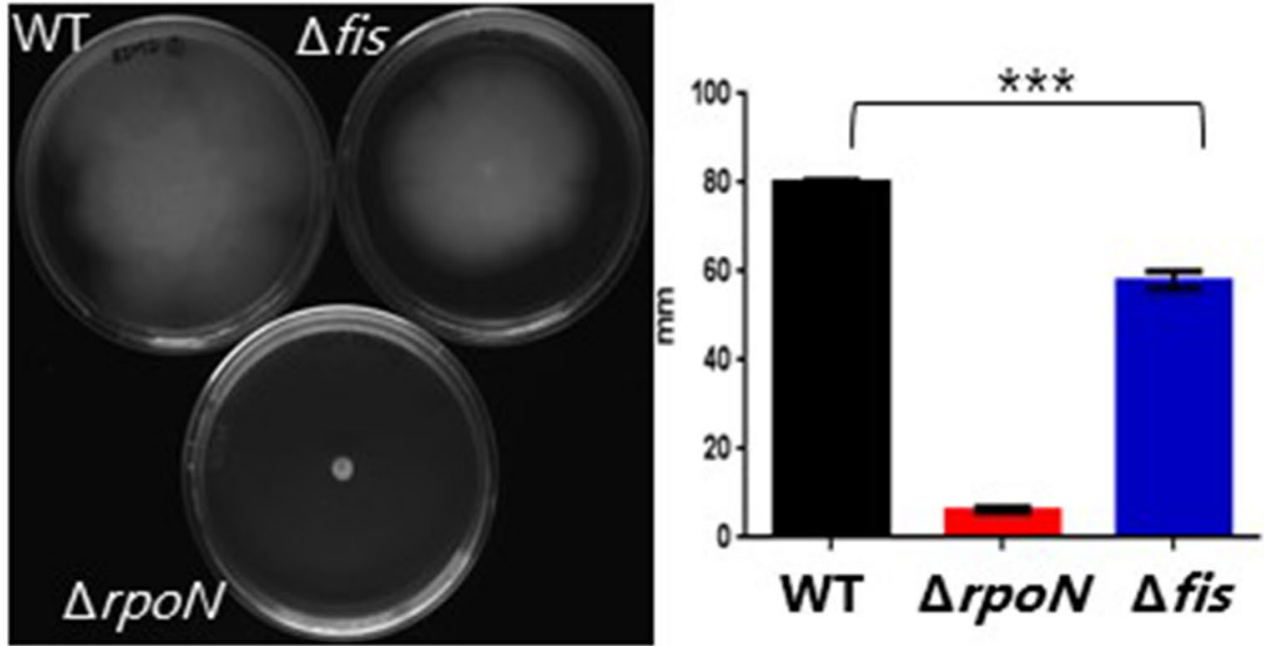

C

\section{Swarming motility assay}
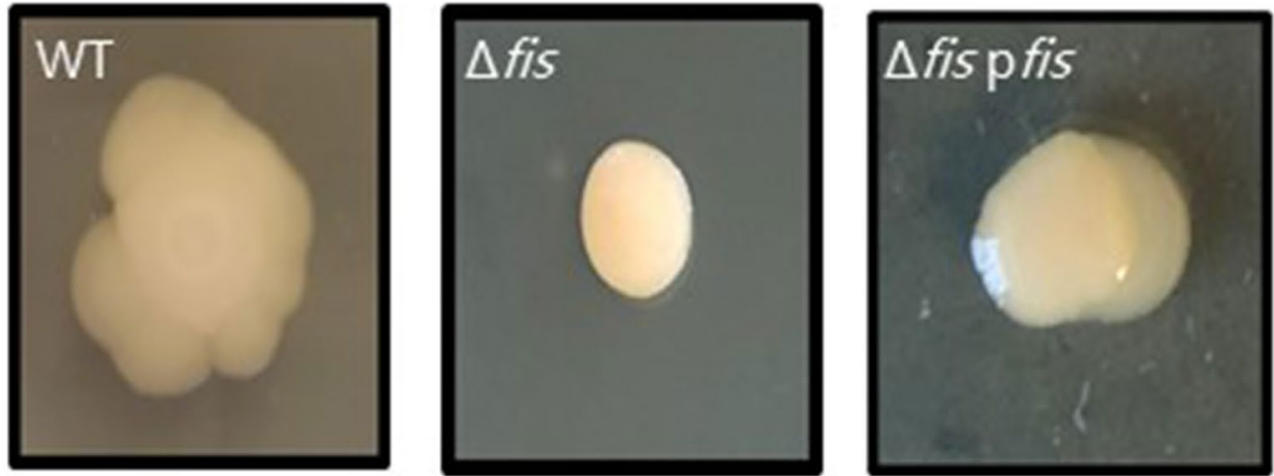

FIGURE 3 | Phenotypic analysis of the $\Delta$ fis mutant. (A) Capsule polysaccharide (CPS) production in wild type (WT), $\Delta$ fis and $\Delta$ opaR mutants was observed after incubation for $48 \mathrm{~h}$ at $30^{\circ} \mathrm{C}$. Images are an example of three biological replicates performed in triplicate. (B) Swimming motility assays and quantification. Two biological replicates were measured, performed in triplicate. Statistics calculated using a Student's $t$-test $\left({ }^{\star \star \star} p<0.001\right)$. (C) Swarming motility assay of V. parahaemolyticus wild type and the $\Delta$ fis mutant and $\Delta$ fis complementation strain. Complementation assays were grown in the presence of Cm and IPTG. All images are examples from three biological replicates. 
A

\section{Polymyxin B disk assay}

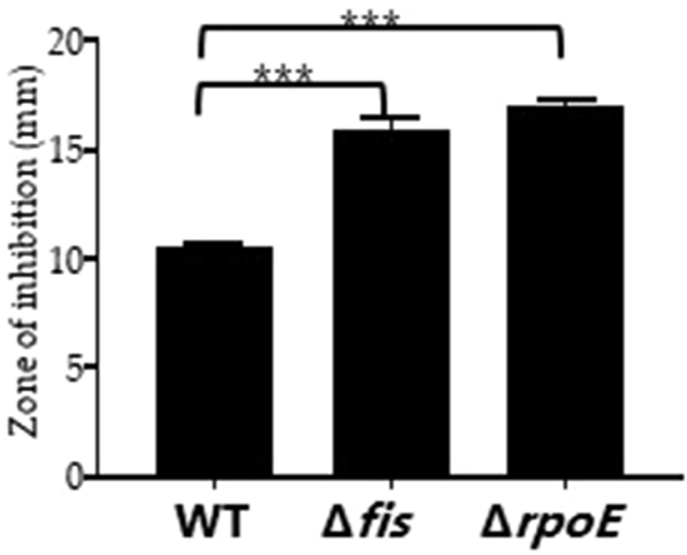

B

\section{Polymyxin B survival assay}

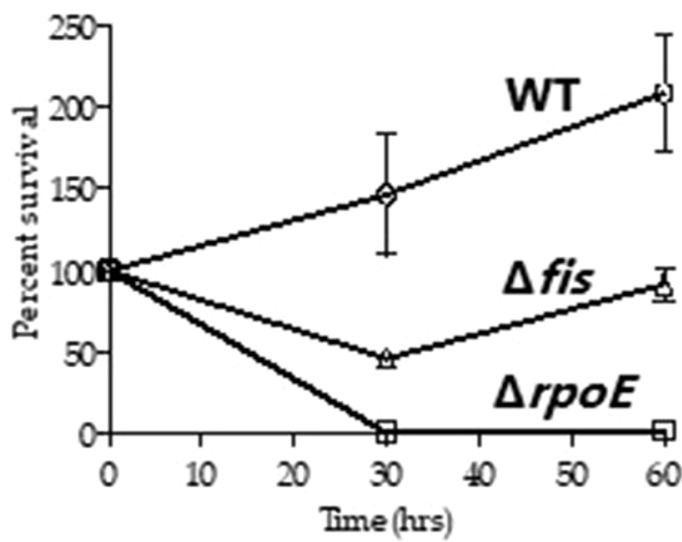

FIGURE 4 | Polymyxin B sensitivity assay. (A) Disk containing $100 \mu \mathrm{g}$ of total polymyxin B was used to identify the zone of inhibition for WT, $\Delta$ fis and $\Delta r p o E$ and was quantified by measuring the diameter of the zone of inhibition. (B) Survival assays were conducted using WT, $\Delta$ fis, and $\Delta r p o E$ after treating the bacterial cultures with polymyxin B (final concentration of $40 \mu \mathrm{g} / \mathrm{ml}$ ) and calculating percent survival at $30 \mathrm{~min}$ and $60 \mathrm{~min}$. The disk assay was performed in duplicate using two biological replicates. The survival assay was performed in triplicate using two biological replicates. Unpaired $t$-test was conducted to determine the value of $p .{ }^{* \star *} p<0.001$.

loci VPA1550-VPA1557) and demonstrated binding (Figures 5B,F). To determine the specificity of Fis binding further, we created two DNA probes with mutations in a single Fis binding motif and examined binding. In these EMSAs, the wild type sequence showed weak binding for both wild type probes and no change in the mutated probe (Figures $5 \mathbf{C , G}$ ). These data suggest that Fis binds at these sites in a non-specific manner. To examine Fis regulation of motility further, GFP reporter expression assays were performed. In these assays, the $\Delta f i s$ mutant relative to wild type did not show differential expression of $\mathrm{P}_{\text {flhA }}-g f p$ (Figure 5D). In contrast, in reporter assays of swarming cells, $\mathrm{P}_{\text {lafB }}-g f p$ was significantly repressed $(p<0.001)$ in the $\Delta$ fis mutant relative to wild type (Figure $5 \mathbf{H})$.
Thus, the data suggest that Fis controls lateral flagella gene expression and is an important positive regulator of swarming motility.

\section{Fis Is a Positive Regulator of the Surface Sensing Operon scrABC}

The $s c r A B C$ operon has been shown to positively control swarming motility and negatively control CPS by controlling c-di-GMP levels (Boles and McCarter, 2002; Kim and McCarter, 2007; Gode-Potratz et al., 2011; Ferreira et al., 2012). We reasoned that Fis might also regulate this operon to co-ordinate with the QS pathway in the control of this phenotype. First, using bioinformatics, we identified multiple and sometimes overlapping putative Fis binding sites in the regulatory region of the $s c r A B C$ operon (Figure 6A). We performed EMSAs with three probes that contained Fis BS and demonstrated Fis binding to all three probes in a concentration dependent manner (Figure 6B). To show Fis BS specificity, we created a DNA probe from probe 3 that contains one Fis BS, mutated this site, and performed an EMSA. In this EMSA, Fis still bound to the mutated probe, but at a reduced level (Figure 6C). In GFP reporter assays, $\mathrm{P}_{\text {scraBC}}-g f p$ activity was significantly downregulated $(p<0.001)$ in the $\Delta$ fis mutant compared to wild type, indicating that Fis is a direct positive regulator of this operon (Figure 6D). Overall, the data suggest that loss of swarming motility is likely due to Fis direct regulation of the $s c r A B C$ operon and the lateral flagella biosynthesis laf operon and indirect regulation of opaR through modulation of $q r r$ expression in $V$. parahaemolyticus.

\section{Fis Modulates Expression of Carbon Catabolism Genes}

To determine the effects of Fis on overall metabolism, we conducted growth competition assays between wild type and $\Delta$ fis in various carbon sources. For the in vitro competition assays, we used a $\beta$-galactosidase knock-in strain of RIMD2210633, strain WBWlacZ, which was previously demonstrated to behave identically to wild type in in vitro and in vivo studies (Whitaker et al., 2012, 2014; HainesMenges et al., 2014; Kalburge et al., 2017). Comparisons of WBWlacZ and the $\Delta$ fis mutant allows for a blue/white colony screen in the presence of IPTG. First, in vitro growth competition assays were performed in $\mathrm{LB} 3 \% \mathrm{NaCl}$ (LBS) resulting in a competitive Index (CI) of 0.99 demonstrating under these conditions that neither strain had a competitive advantage. Similarly, in vitro competition assays in M9 $3 \% \mathrm{NaCl}$ (M9S) supplemented with glucose showed neither strain had a competitive advantage. However, in M9S supplemented with mouse intestinal mucus or individual mucus components as sole carbon sources, $\Delta$ fis was significantly outcompeted by WBWlacZ. In mouse intestinal mucus, the $\Delta$ fis mutant had a CI of 0.61 , and in mucus components L-arabinose a CI of 0.4 , NAG a CI of 0.68 and D-gluconate a CI of 0.78 (Figure 7).

Putative Fis binding sites were identified in the regulatory region of the araBDAC (L-arabinose catabolism) operon 
A

fl $h$ polar gene cluster

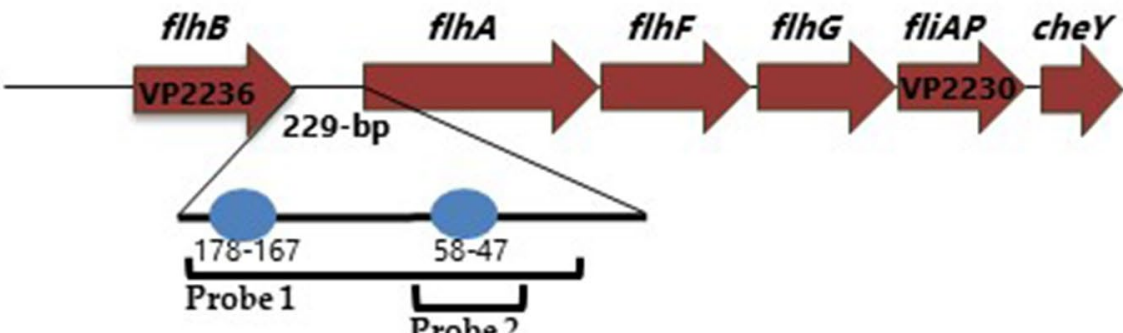

B

EMSA probe 1

[Fis]

$1: 0 \quad 1: 1 \quad 1: 10 \quad 1: 20$ c

EMSA probe 2

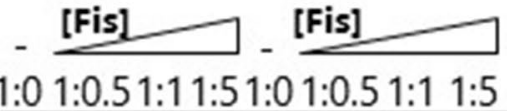

1:0 1:0.51:11:51:0 1:0.5 1:1 1:5

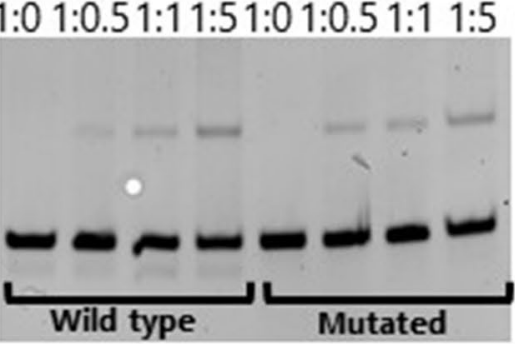

D

Reporter assay P flhA-gfp

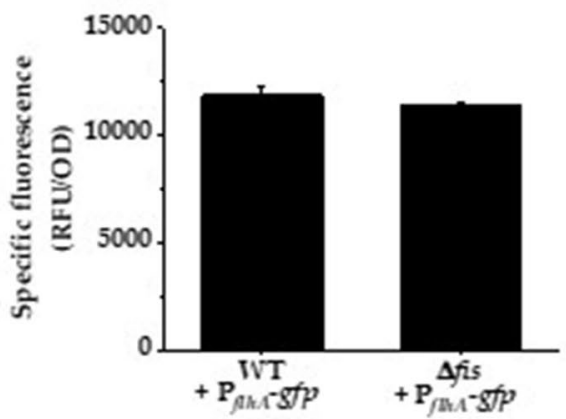

E

fla lateral gene cluster

lafA hp lafB lafC lafD lafE lafL fliAL laft lafU

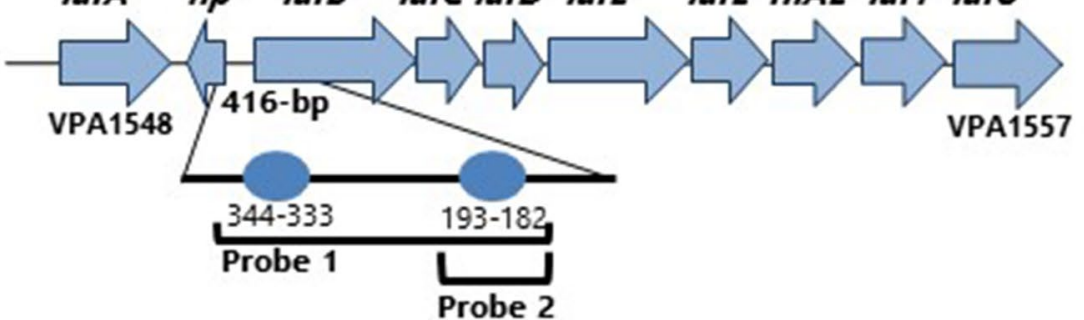

F

\section{EMSA probe 1}

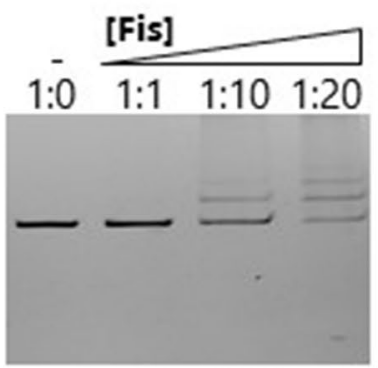

Probe 1
G

EMSA probe 2

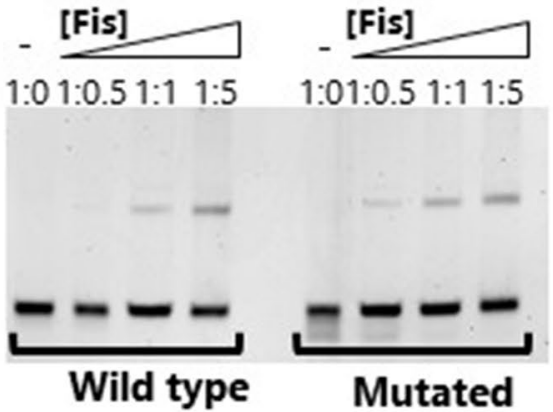

H

\section{Reporter assay PlafB-gfp}

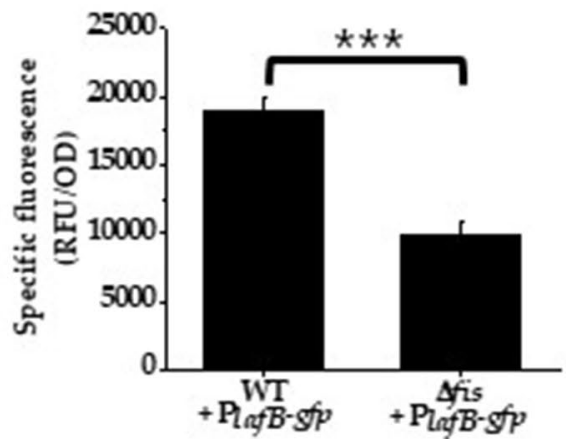

FIGURE 5 | Regulation of lateral flagella biosynthesis by Fis. (A) Regulatory region of polar flagellum fih genes with putative Fis binding sites (BS) depicted as blue circles. (B) EMSA of Fis bound to PflhA in a concentration dependent manner. DNA: protein ratios are as follows: 10, 1:1, 1:10, 1:20. (C) Probe 2 containing a single Fis BS (WT) was mutated. DNA: protein ratios are as follows: 0, 1:0.5, 1:1, 1:5. (D) Transcriptional GFP reporter assay of PflhA-gfp in the $\Delta$ fis mutant relative to wild type. (E) Regulatory region of lateral flagellum laf genes with putative Fis binding sites. (F) EMSA of Fis bound to Plaf DNA probe. (G) Probe 2 containing a single Fis BS and a mutated probe 2 Fis BS. DNA: protein ratios are as follows: 0, 1:0.5, 1:1, 1:5. (H) Transcriptional GFP reporter assay of PlafB-gfp between wild type and $\Delta$ fis $\left({ }^{\star \star \star} p<0.001\right)$. 
A

$s c r A B C$ regulatory region

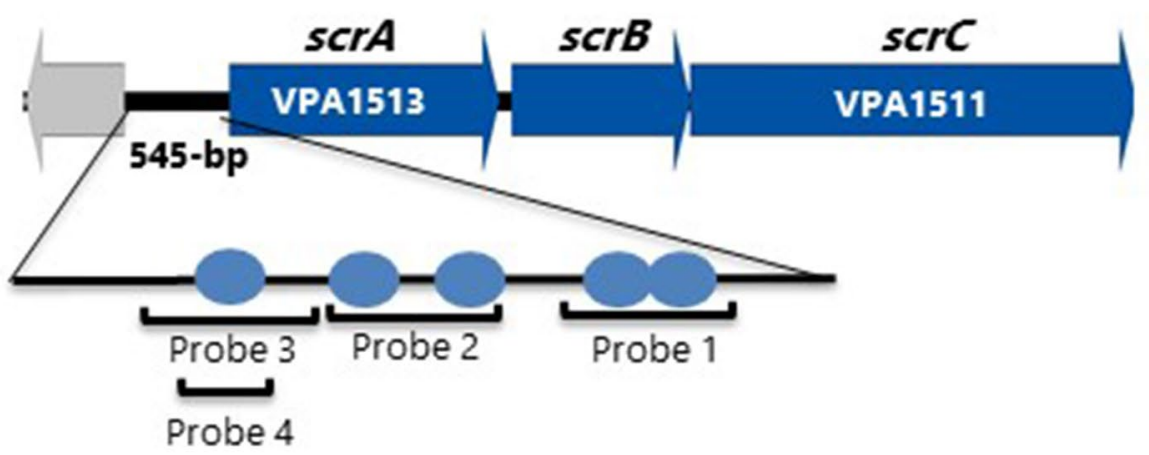

B

EMSA analysis PscrABC

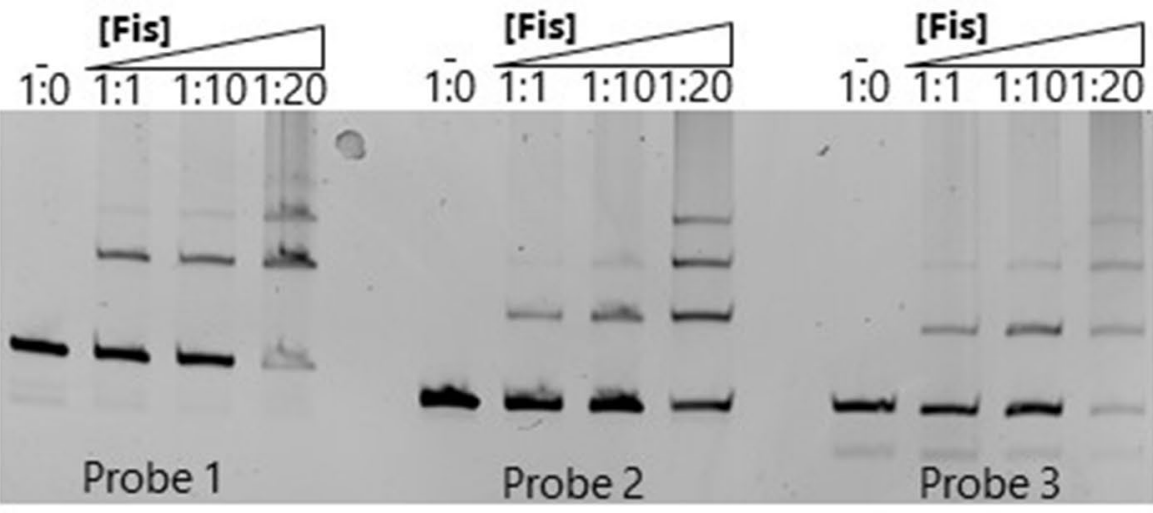

C

EMSA probe 3

${ }^{\mathrm{D}}$ Reporter assay PscrABC-gfp

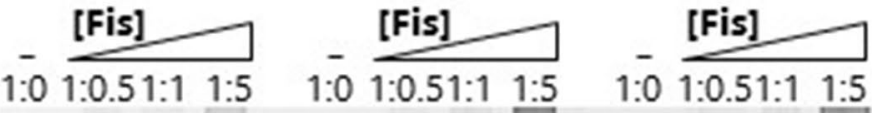

1:0 1:0.51:1 $1: 5$

1:0 1:0.51:1 1:5

1:0 1:0.51:1 1:5

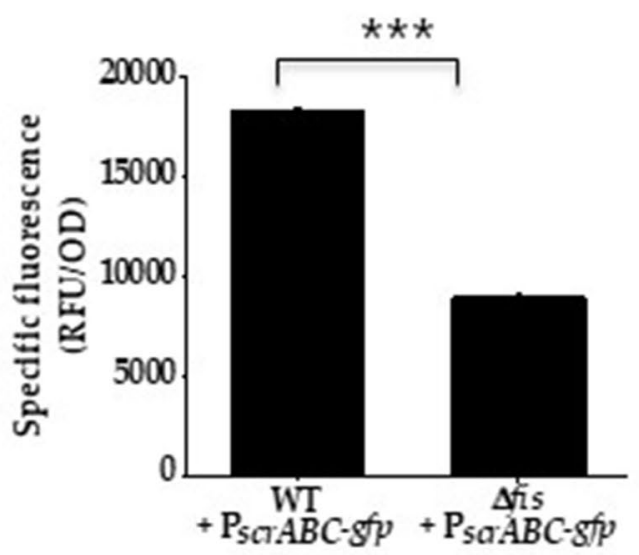

FIGURE 6 | Fis is a positive regulator of the surface sensing operon, scrABC. (A) Putative Fis binding sites identified in the regulatory region of the $S C r A B C$ surface sensing operon. (B) EMSA using purified Fis protein and probes 1, 2 and 3 encompassing the regulatory region of SCrABC. DNA: protein ratios are as follows: 10, 1:1, 1:10, 1:20, 1:50. (C) Probe 3 containing a single Fis BS and a mutated probe 3 Fis BS. DNA: protein ratios are as follows: 0, 1:0.5, 1:1, 1:5. (D) GFP reporter assay of PscrABC-gfp in wild type and the $\triangle$ fis mutant. Specific fluorescence was calculated (RFU/OD) for three biological replicates and plotted as mean and standard deviation. Statistics were calculated using a Student's $t$-test $\left({ }^{\star \star \star} p<0.001\right)$.

(Figure 8A). EMSAs were performed using 138-bp and 152-bp DNA probes containing two and one putative Fis binding sites, respectively (Figure $\mathbf{8 A}$ ). In these assays, Fis bound to the regulatory region of $a r a B D A C$ and the binding was concentration dependent (Figure 8B). To examine the specificity of Fis binding further, we created probe 3 that contained a single Fis BS, 


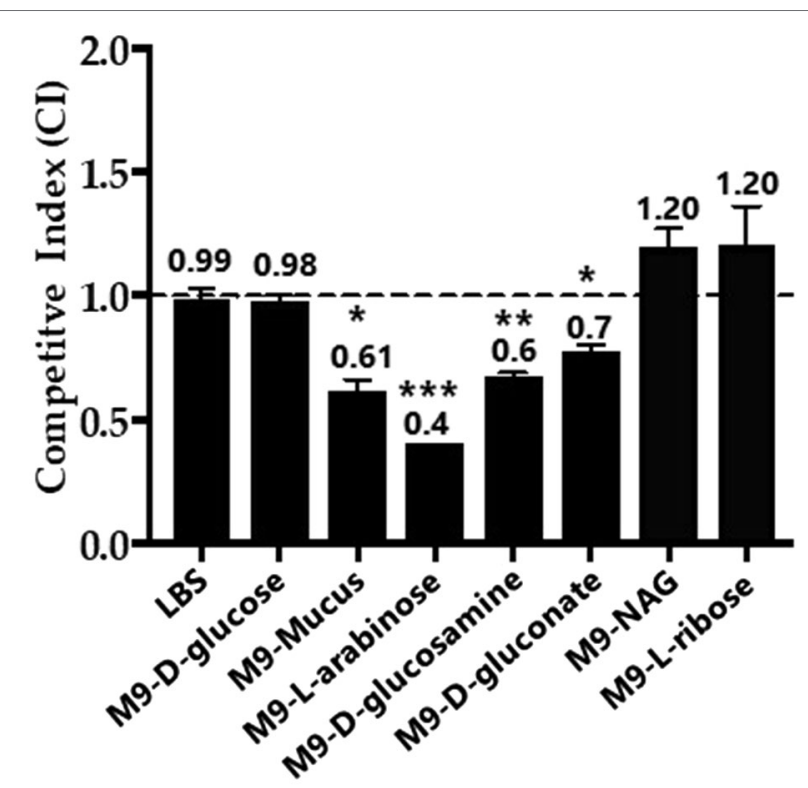

FIGURE 7 | In vitro growth competition assay between wild type and the $\Delta$ fis mutant. WBWlacZ and $\Delta$ fis were grown in co-culture (1:1 ratio) for $24 \mathrm{~h}$ in LBS, M9 supplemented with $100 \mu \mathrm{g} / \mathrm{ml}$ intestinal mucus, $10 \mathrm{~mm}$ of D-glucose, L-arabinose, D-glucosamine, D-gluconate, $\mathrm{N}$-acetyl-D-glucosamine (NAG), or D-ribose. WBWlacZ outcompetes $\Delta$ fis, $<1.00$, and $\Delta$ fis outcompetes WBWlacZ when $>1.00$. The assay was conducted in two biological replicates in triplicates. Error bar indicates SEM. Unpaired Student's $t$-test was conducted. The significant difference is denoted by asterisks $\left({ }^{*} p<0.05\right.$, $\left.{ }^{\star *} p<0.01,{ }^{\star * \star} p<0.001\right)$.

mutated this site, and performed an EMSA. The wild type probe 3 showed Fis binding, whereas the mutated probe showed no binding, demonstrating Fis binding specificity (Figure 8C). In GFP transcriptional reporter assays, $\mathrm{P}_{\text {araB }}-g f p$ showed lower activity in the $\Delta$ fis mutant compared to wild type, although the fold change difference is small suggesting it may not be physiologically relevant at this time-point (Figure 8D).

Factor for inversion stimulation binding sites were also identified in the regulatory region of gntK (D-gluconate catabolism; Figure 9A). A DNA probe encompassing PgntK showed Fis binding (Figure 9B). A second probe with only a single putative Fis BS was constructed and the putative Fis BS was mutated and EMSAs performed. In this assay a slight reduction in binding is noted, but non-specific binding cannot be ruled out (Figure 9C). To examine Fis control of D-gluconate metabolism further, a GFP reporter assay of PgntK was performed. In these assays, PgntK showed significantly lower expression levels in the $\Delta$ fis mutant compared to wild type indicating that Fis is a positive regulator of expression (Figure 9D).

Multiple overlapping Fis binding sites were identified in the regulatory region of $n a g B$ (NAG catabolism; Figure 10A). EMSA analysis with two probes encompassing the Fis sites showed specific binding in a concentration dependent manner (Figures 10B,C). To examine specificity of binding further we created a probe 2 with mutations in 5 sites of the Fis BS motif. In this analysis, Fis bound to the mutated probe, but the binding was reduced, suggesting Fis is a direct regulator of nagB (Figure 10C). In addition, PnagB showed significantly lower activity in the GFP reporter assay in the $\Delta$ fis mutant compared to wild type (Figure 10D). Overall, our results demonstrated that $\mathrm{Fis}$ is a positive regulator of catabolism genes, araB, gnt $K$, and $n a g B$ in $V$. parahaemolyticus.

We determined a Fis consensus sequence from the regulatory regions of genes under Fis control. To accomplish this, we examined a 50-bp region encompassing each putative Fis binding site identified in this study using multiple EM for motif elicitation (MEME) analysis. This analysis identified a 15-bp core motif highly similar to what has been identified in E. coli (Figure 11A). This motif is aligned in each sequence and maps to the Fis binding sites identified via virtual footprint (Figure 11B). Similar to the sequence motif used by virtual footprint, along with previous reports of Fis consensus sequences, the motif we present has a stretch of T/A bps, characteristic of Fis binding (Cho et al., 2008).

\section{Fis Is Required for in vivo Fitness in V. parahaemolyticus}

To determine whether Fis contributes to in vivo fitness of $V$. parahaemolyticus, in vivo colonization competition assays were performed using a streptomycin pretreated adult mouse model of colonization (Whitaker et al., 2012, 2014; Haines-Menges et al., 2014; Yang et al., 2019). The in vivo competition assay determined the ability of WBWlacZ and the $\Delta$ fis mutant to co-colonize the intestinal tract of streptomycin pretreated adult mice. Competition assays were performed in adult C57BL/6 mice pretreated with an orogastric dose of streptomycin $(20 \mathrm{mg} /$ animal) $24 \mathrm{~h}$ prior to orogastric co-inoculation with a $1: 1$ mixture of $V$. parahaemolyticus WBWlacZ and $\Delta$ fis $(n=9)$. In an in vitro assay in LBS using the same inoculum, the WBWlacZ vs. $\Delta$ fis had a CI of 1.17 whereas in vivo the WBWlacZ vs. $\Delta$ fis CI was 0.57 indicating that the mutant was significantly $(p<0.01)$ outcompeted by WBWlacZ (Supplementary Figure S4). This indicates that $\Delta$ fis has decreased fitness in vivo compared to the wild type strain.

\section{DISCUSSION}

Nucleoid associated proteins such as Fis, bind and bend DNA to aid in DNA compaction, and are also important global regulators of gene expression. In $V$. parahaemolyticus we show, similar to enteric species, that fis is expressed in early to mid-exponential phase cells and declines in late exponential and stationary phase cells. The only other study examining the role of Fis in a Vibrio species, showed a direct role of Fis in the QS pathway in V. cholerae. They demonstrated Fis activation of qrr1 to qrr4 sRNAs and hapR (opaR homolog) constitutive expression in a fis deletion mutant (Lenz and Bassler, 2007). In V. parahaemolyticus, Fis bound to all five qrr sRNAs regulatory regions in a concentration dependent manner. Our work showed that Fis is a positive regulator of the QS regulatory sRNAs. We show that in the fis deletion mutant qrr1 to qrr5 were repressed and $o p a R$ was induce. It 
A

L-arabinose metabolism gene cluster

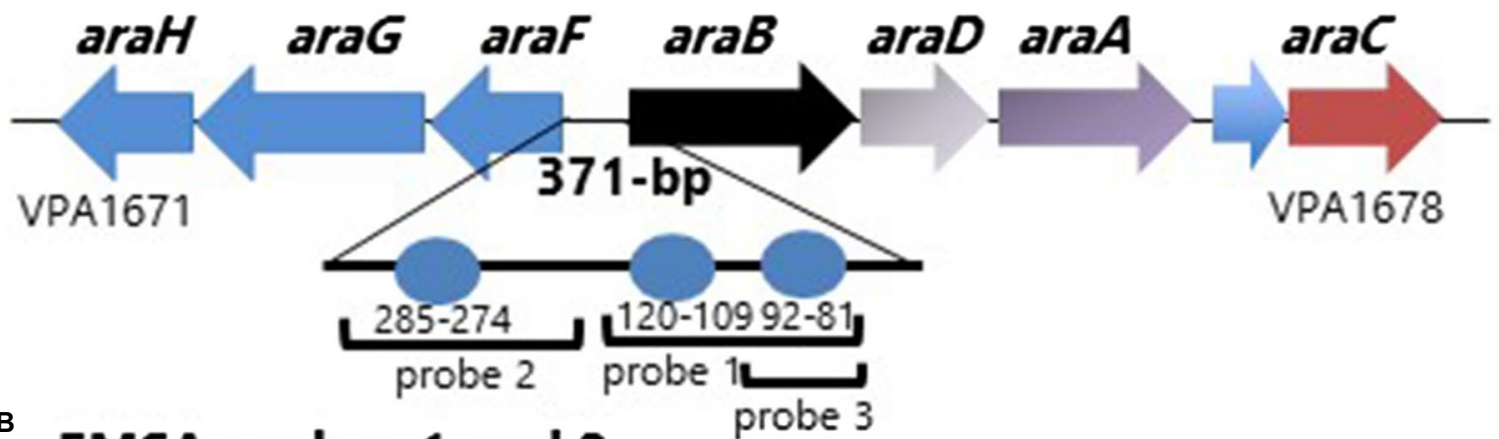

B EMSA probes 1 and 2

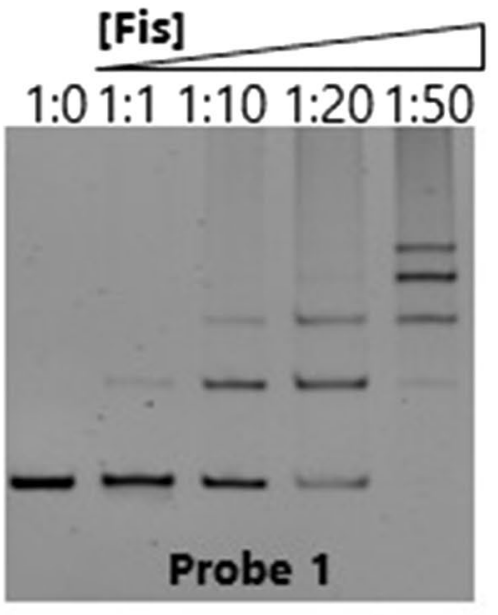

C
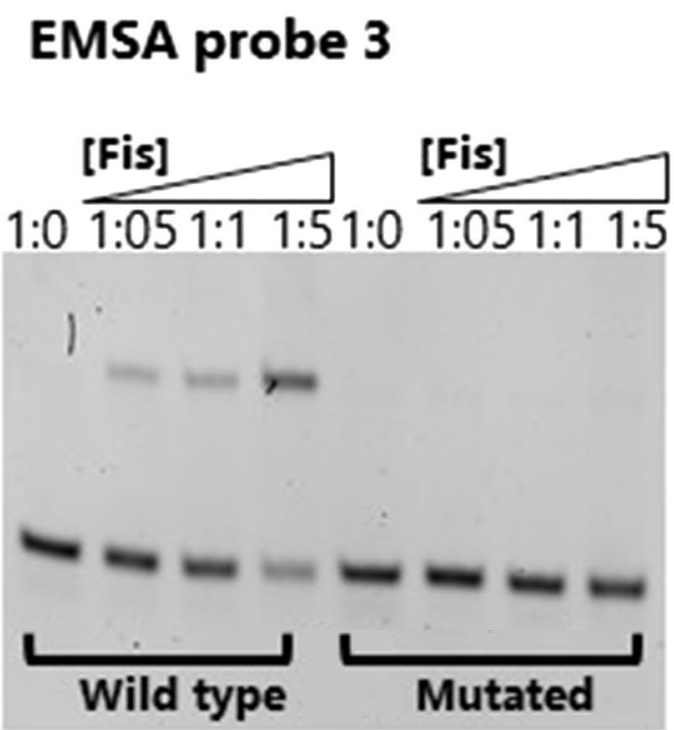

[Fis]

1:0 1:1 1:10 1:201:50

Probe 2

D

Reporter assay ParaB-gfp

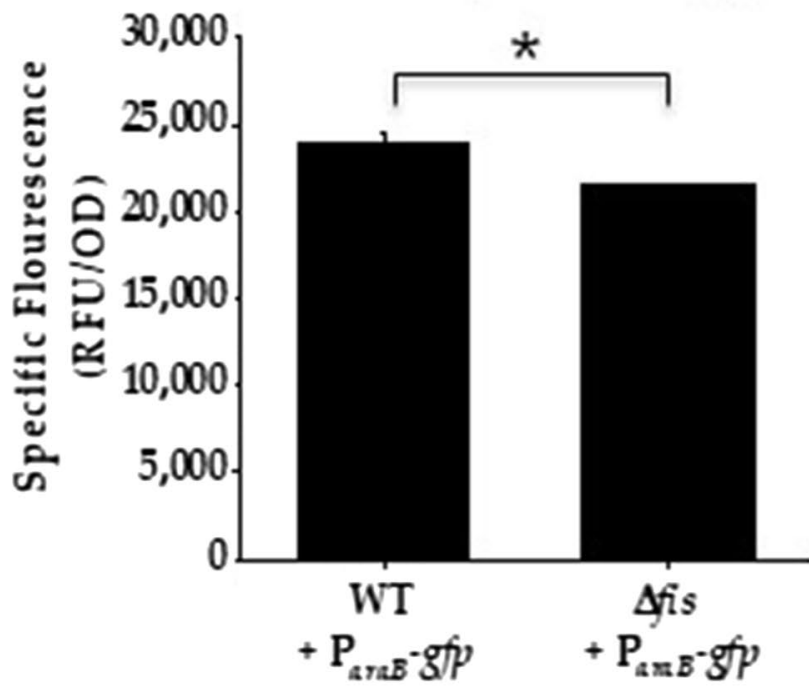

FIGURE 8 | Fis is a positive regulator of the L-arabinose operon araBCDA. (A) Fis binding sites identified in the regulatory region of the araBDAC operon depicted as blue circles. (B) ParaB was divided into two probes, probe 1 and probe 2 for EMSA analysis using purified Fis protein. (C) EMSA analysis probe 3 wild type with a single Fis BS and a mutated probe 3. DNA: protein ratios were as follows: 10, 1:1, 1:10, 1:20, 1:50. (D) GFP transcriptional reporter assay of the araBDAC regulatory region in wild type and the $\Delta$ fis mutant. ${ }^{*} p<0.05$. 
A

D-Gluconate metabolism gene cluster

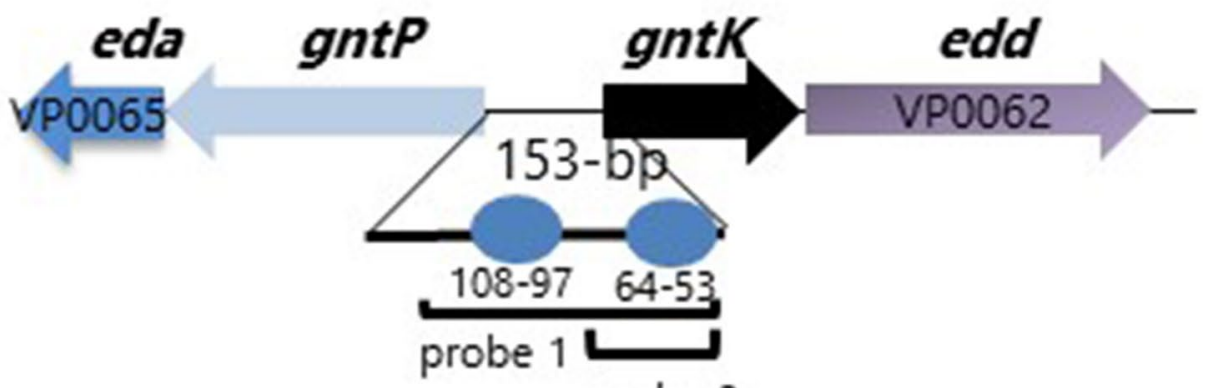

B

EMSA PgntK

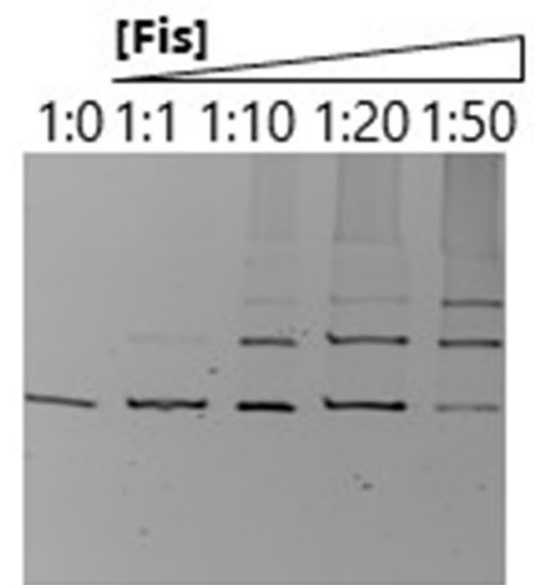

c

EMSA probe 2

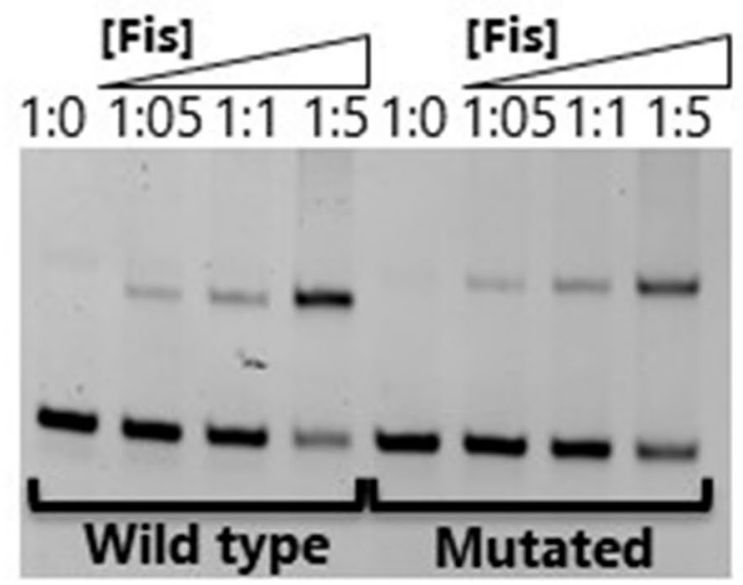

\section{D}

Reporter assay PgntK-gfp

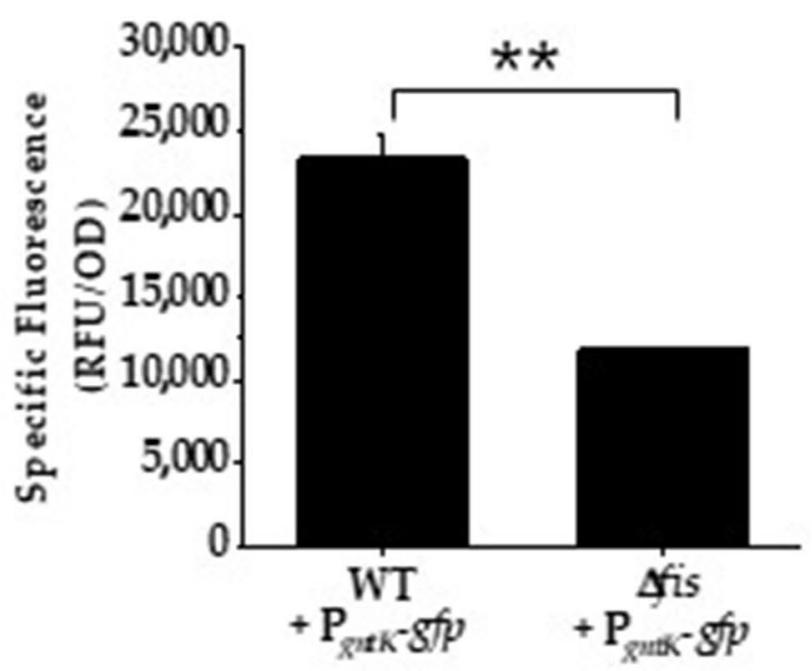

FIGURE 9 | Fis is a positive regulator of gntK. (A) Putative Fis binding sites in the regulatory region of gntK. (B) EMSA analysis with purified Fis protein and PgntK probe 1. DNA: protein ratios were as follows: 10, 1:1, 1:10, 1:20, 1:50. (C) EMSA analysis of probe 2 containing a single Fis BS and a mutated Fis BS probe 2. (D) GFP transcriptional reporter assay of gntK regulatory region in wild type and $\Delta$ fis. ${ }^{* *} p<0.01$. 
A

N-acetyl-D-glucosamine-P (NagB)

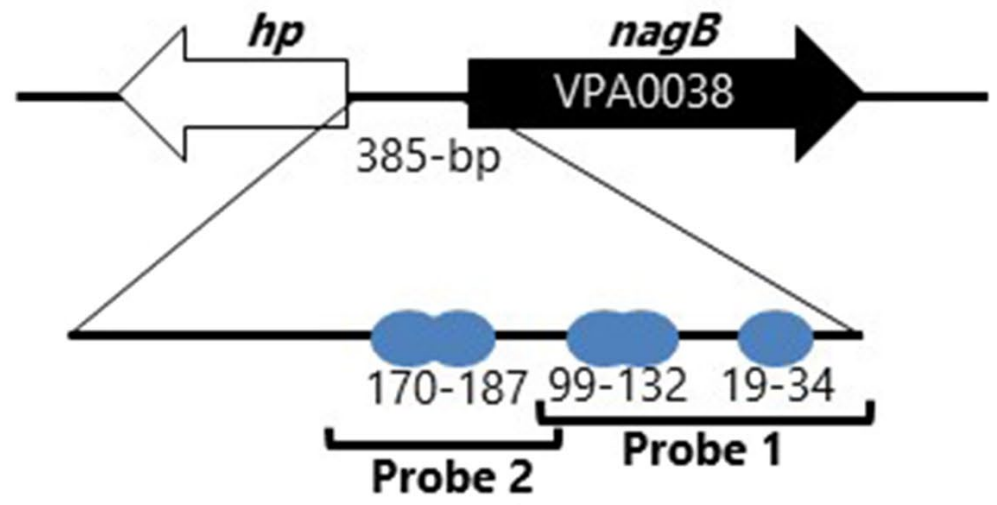

B

EMSA probe $1^{\mathrm{C}}$ EMSA probe 2

[Fis]
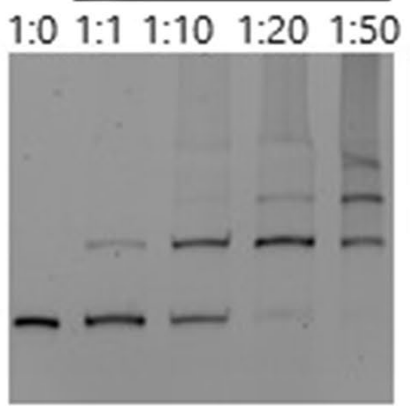

D

\section{GFP reporter assay}

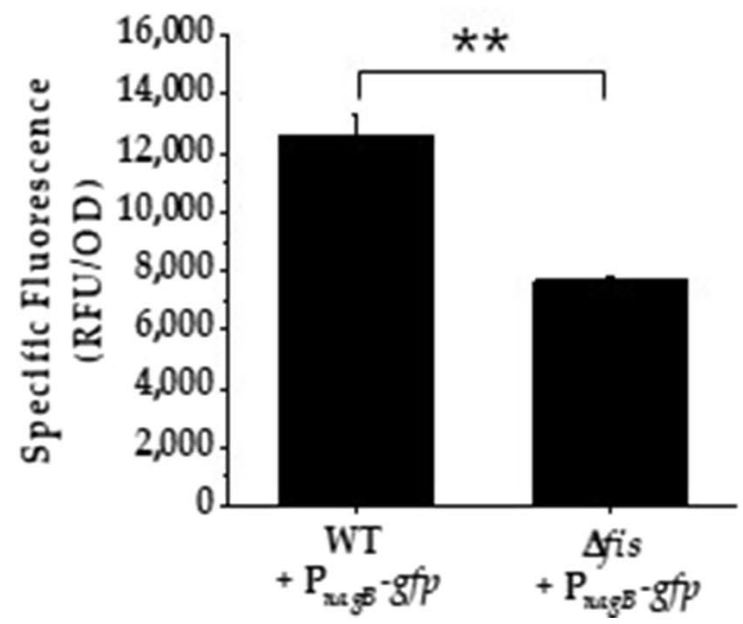

FIGURE 10 | Fis is a positive regulator of NAG gene nagB. (A) Fis binding sites identified in the regulatory region of nagB. (B) EMSA analysis of Fis binding to probe 1. (C) EMSA analysis of probe 2 and a mutated Fis BS probe 2. DNA: protein molar ratios were as follows: 10, 1:1, 1:10, 1:20, 1:50. (D) GFP transcriptional reporter assay with PnagB-gfp. Means and standard deviations of two biological replicates are shown. Statistics calculated using a Student's $t$-test $\left({ }^{* *} p<0.01\right)$. 


\begin{tabular}{|c|c|c|}
\hline Region & P-value & Binding Sites \\
\hline Pqrr2_A & $2.21 e-5$ & atgagatctt GTCCCCAAAAATAAA gctttgaaaa \\
\hline Pqrr2_C & $2.35 e-5$ & aaatgttaac GAGTTAAGGTTC C tgttctcgco \\
\hline PlafB_A & $5.11 \mathrm{e}-7$ & aaaatatgce GAGTTAAAA ATACC aaaaatcgat \\
\hline PlafB_C & $2.06 e-5$ & aagacaaggc GACTAAAAAGCT AA aatatttaat \\
\hline PscrA_A & $8.27 e-7$ & cttaatggca GAGTAAAAAATTAAA g \\
\hline PscrA_B & $2.06 e-5$ & gttataaatt CATTAAAAATTTAAC tttgtattaa \\
\hline PscrA_C & $5.03 e-6$ & cgctatatat GGTCTAAAAATTAAC tgttcattta \\
\hline PgyrA & $8.27 e-7$ & tacgtgtaag GTGCAAAAATATAAC acacaattca \\
\hline
\end{tabular}

B

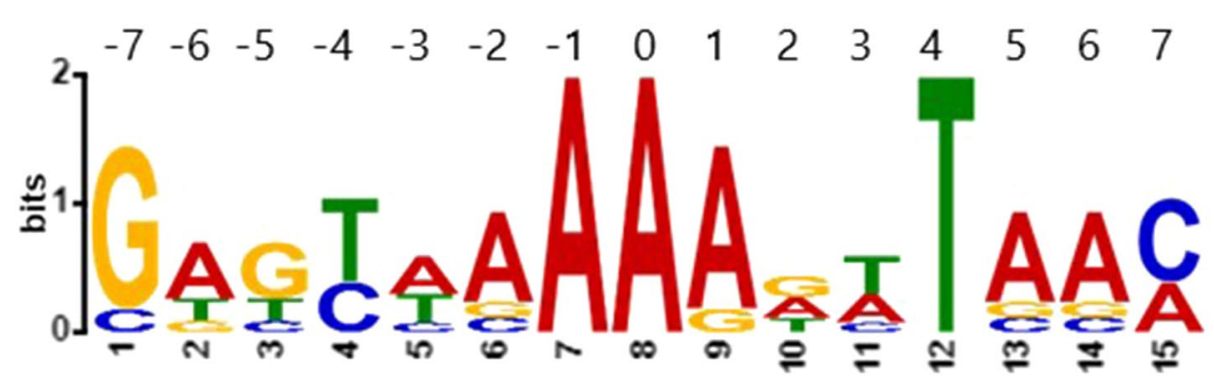

FIGURE 11 | Analysis of Fis consensus sequence. (A) A Fis DNA binding motif was created using MEME analysis. Sequences containing a putative Fis binding site were used. (B) An alignment of the motifs found in each sequence, along with the corresponding values of $p$.

is of interest to note that in our study, the expression of each of the qrr sRNA differed in the $\Delta$ fis mutant compared to wild type with significant repression of $q r r 2$, qrr3 and qrr4, whereas both $q r r 1$ and $q r r 5$ showed reduced expression. The importance of this remains to be determine since we do not know whether in V. parahaemolyticus the Qrr sRNAs are redundant as is the case in $V$. cholerae or additive as is the case in $V$. harveyi. Our data suggest that other regulators are also involved in modulating qrr expression and that Fis is one of several factors controlling expression. This is not too surprising as studies have shown that, although Fis may bind to a large number of regulatory regions, only a portion of these sites are significantly regulated by Fis (Kahramanoglou et al., 2011; Monteiro et al., 2020). For example, in E. coli, ChIP-seq analysis uncovered 1,464 Fis binding sites, but only 462 genes were differential regulated by Fis under the conditions examined (Kahramanoglou et al., 2011). This suggests that Fis has a regulatory role, however other factors are likely involved and that the Fis regulon varies with changes in growth conditions, growth phase, binding affinity, amongst other factors.

In $V$. parahaemolyticus, we showed that $F$ is is a positive regulator of swarming motility through modulated expression of the QS regulator, OpaR, and control of the lateral flagellum laf operon and the surface sensing $s c r A B C$ operon. The $s c r A B C$ operon in $V$. parahaemolyticus controls the transition between swarming motility and adhesion to a surface by altering gene expression of the laf and cps operons (Boles and McCarter, 2002; Kim and McCarter, 2007). Together, ScrA, ScrB, and
ScrC modulate the level of c-di-GMP in the cell, a secondary messenger that controls numerous downstream processes. More specifically, ScrC contains both GGDEF and EAL enzymatic activity, making it a unique bifunctional enzyme (Kim and McCarter, 2007; Ferreira et al., 2012). ScrA produce the extracellular S-signal, which represses CPS gene expression. In the presence of ScrA interaction with ScrB, ScrC acts as a phosphodiesterase to degrade c-di-GMP. High levels of c-di-GMP promote CPS production, while low levels of c-di-GMP promote swarming motility (Trimble and McCarter, 2011; Ferreira et al., 2012). In the $\Delta$ fis mutant, we observed repression of both the laf and $s c r A B C$ operons in the GFP reporter assays, indicating that $F$ is is a positive regulator of these operons. We suggest that the $\Delta$ fis mutant swarming defect is through repression of both the laf and $s c r A B C$ operons and through derepression of $o p a R$, which is a repressor of swarming motility (Figure 12). As shown in Supplementary Figure S1, fis expression is induced at early exponential phase cells and decreases as the cells transition to HCD. We propose that at LCD, Fis activates the qrr sRNAs, which repress $o p a R$, and activates the surface sensing operon. The scrABC operon activates the laf operon, stimulating motility, while repressing the cps operon. As the cells transition to HCD, fis expression decreases while opaR expression increases, as it has been shown to be maximally expressed in stationary phase cells. OpaR represses the laf operon and activates expression of the cps operon, inducing CPS formation. Therefore, Fis integrates both the QS and surface sensing 


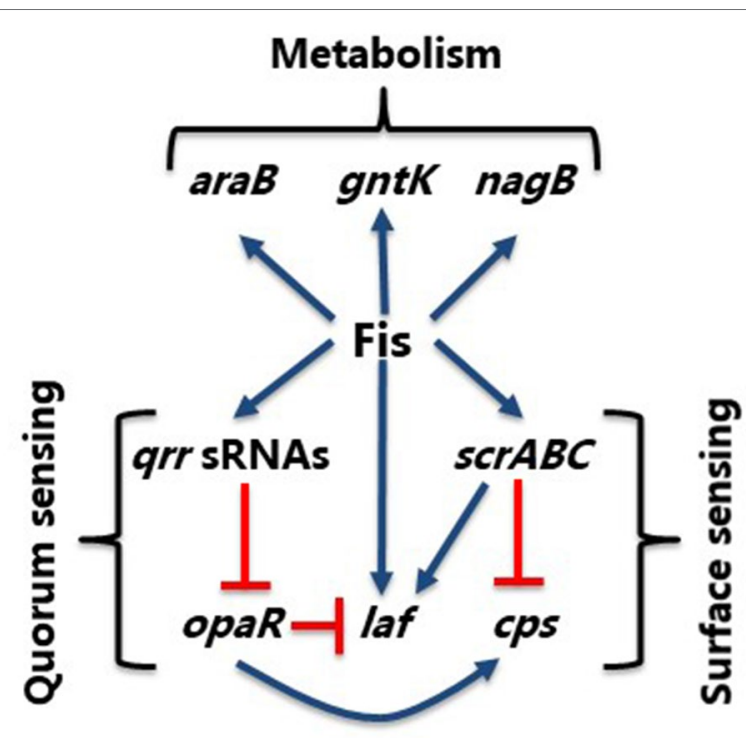

FIGURE 12 | Model of Fis integration of QS and surface sensing pathways. Model shows control of swarming (laf) and CPS (cps) production in $V$. parahaemolyticus. Blue arrows show positive regulation and orange hammers show negative regulation.

signals by positively regulating $q r r$ sRNAs and $s c r A B C$ to induce swarming motility and repress CPS formation until the cells enter HCD (Figure 12).

In $E$. coli, using global approaches, Fis was shown to regulate genes involved in, but not limited to, two-component systems, biofilm formation, and pilus organization. It was determined that Fis regulates genes required for sugar metabolism as well, including NAG as shown in our study (Gawade et al., 2020). In S. enterica, Fis was shown to negatively regulate genes contributing to virulence and metabolism in the mammalian gut (Kelly et al., 2004). In this species, it was demonstrated that Fis was a negative regulator of acetate metabolism, biotin synthesis, fatty acids metabolism and propanediol utilization, amongst others. The authors speculated that this could be important for intestinal colonization and/or systemic infection (Kelly et al., 2004). In V. parahaemolyticus, the in vivo competition assays between the $\Delta$ fis and WBWLacZ strain showed that the $\Delta$ fis strain was outcompeted demonstrating that Fis is required for in vivo fitness. In addition, in vitro growth competition assays in intestinal mucus and mucus components demonstrated that $\Delta f i s$ was again outcompeted by wild type. We speculate that the $\Delta$ fis mutant is outcompeted by wild type in vivo at least in part due to its inability to efficiently utilize nutrient sources. Carbon metabolism was previously implicated as important for colonization of $V$. parahaemolyticus in a streptomycin pretreated adult mouse model (Whitaker et al., 2014;

\section{REFERENCES}

Auner, H., Buckle, M., Deufel, A., Kutateladze, T., Lazarus, L., Mavathur, R., et al. (2003). Mechanism of transcriptional activation by FIS: role of core promoter structure and DNA topology. J. Mol. Biol. 331, 331-344. doi: $10.1016 / \mathrm{S} 0022-2836(03) 00727-7$
Kalburge et al., 2017). Additionally, we showed that Fis is important for resistance to antimicrobial peptides prevalent in animal intestines. The fis deletion mutant was highly sensitive to polymyxin $\mathrm{B}$, which could also account for its reduced fitness in vivo. In summary, Fis modulates expression of genes involved in QS, motility, and metabolism in V. parahaemolyticus and it will be of interest to determine the different mechanisms used to modulate expression by this NAP.

\section{DATA AVAILABILITY STATEMENT}

The original contributions presented in the study are included in the article/Supplementary Material, further inquiries can be directed to the corresponding author.

\section{ETHICS STATEMENT}

The animal study was reviewed and approved by University of Delaware Institutional Animal Care and Use Committee.

\section{AUTHOR CONTRIBUTIONS}

$\mathrm{EB}, \mathrm{JT}$, and AR designed the study and analyzed the results. JT, GG, and AR performed the experiments. All authors contributed to writing and review the manuscript and approved the submitted version.

\section{FUNDING}

This research was supported in part by a National Science Foundation grant (award IOS-1656688) to EB. JT and GG were funded by University of Delaware graduate fellowship awards.

\section{ACKNOWLEDGMENTS}

We thank members of the Boyd Group for constructive feedback on the manuscript and the reviewers for their suggestions and comments.

\section{SUPPLEMENTARY MATERIAL}

The Supplementary Material for this article can be found online at: https://www.frontiersin.org/articles/10.3389/fmicb.2021.669447/ full\#supplementary-material

Azam, T. A., Iwata, A., Nishimura, A., Ueda, S., and Ishihama, A. (1999). Growth phase-dependent variation in protein composition of the Escherichia coli nucleoid. J. Bacteriol. 181, 6361-6370. doi: 10.1128/JB.181.20.63616370.1999

Bassler, B. (1999). How bacteria talk to each other: regulation of gene expression by quorum sensing. Curr. Opin. Microbiol. 2, 582-587. doi: 10.1016/ S1369-5274(99)00025-9 
Bassler, B. L., Greenberg, E. P., and Stevens, A. M. (1997). Cross-species induction of luminescence in the quorum-sensing bacterium Vibrio harveyi. J. Bacteriol. 179, 4043-4045. doi: 10.1128/jb.179.12.4043-4045.1997

Belas, R., Simon, M., and Silverman, M. (1986). Regulation of lateral flagella gene transcription in Vibrio parahaemolyticus. J. Bacteriol. 167, 210-218. doi: $10.1128 / \mathrm{jb} \cdot 167.1 .210-218.1986$

Bétermier, M., Galas, D., and Chandler, M. (1994). Interaction of Fis protein with DNA: bending and specificity of binding. Biochimie 76, 958-967. doi: 10.1016/0300-9084(94)90021-3

Bokal, A. J., Ross, W., Gaal, T., Johnson, R. C., and Gourse, R. L. (1997). Molecular anatomy of a transcription activation patch: FIS-RNA polymerase interactions at the Escherichia coli $\mathrm{rnB}$ P1 promoter. EMBO J. 16, 154-162. doi: 10.1093/emboj/16.1.154

Boles, B. R., and McCarter, L. L. (2002). Vibrio parahaemolyticus scrABC, a novel operon affecting swarming and capsular polysaccharide regulation. J. Bacteriol. 184, 5946-5954. doi: 10.1128/JB.184.21.5946-5954.2002

Bradley, M. D., Beach, M. B., de Koning, A. P., Pratt, T. S., and Osuna, R. (2007). Effects of Fis on Escherichia coli gene expression during different growth stages. Microbiology 153, 2922-2940. doi: 10.1099/mic.0.2007/008565-0

Browning, D. F., Grainger, D. C., Beatty, C. M., Wolfe, A. J., Cole, J. A., and Busby, S. J. (2005). Integration of three signals at the Escherichia coli nrf promoter: a role for Fis protein in catabolite repression. Mol. Microbiol. 57, 496-510. doi: 10.1111/j.1365-2958.2005.04701.x

Carpenter, M., Rozovsky, S., and Boyd, E. (2015). Pathogenicity island cross talk mediated by recombination directionality factors facilitates excision from the chromosome. J. Bacteriol. 198, 766-776. doi: 10.1128/JB.00704-15

Cho, B., Knight, E., Barrett, C., and Palsson, B. (2008). Genome-wide analysis of Fis binding in Escherichia coli indicates a causative role for A-/AT-tracts. Genome Res. 18, 900-910. doi: 10.1101/gr.070276.107

Cróinín, T. O., Carroll, R. K., Kelly, A., and Dorman, C. J. (2006). Roles for DNA supercoiling and the Fis protein in modulating expression of virulence genes during intracellular growth of Salmonella enterica serovar Typhimurium. Mol. Microbiol. 62, 869-882. doi: 10.1111/j.1365-2958.2006.05416.x

De Souza Santos, M., and Orth, K. (2019). The role of the type III secretion system in the intracellular lifestyle of enteric pathogens. Microbiol. Spectr. 7. doi: $10.1128 /$ microbiolspec.BAI-0008-2019

Dehio, C., and Meyer, M. (1997). Maintenance of broad-host-range incompatibility group P and group Q plasmids and transposition of Tn5 in Bartonella henselae following conjugal plasmid transfer from Escherichia coli. J. Bacteriol. 179, 538-540. doi: 10.1128/jb.179.2.538-540.1997

Dennis, P. P., Ehrenberg, M., and Bremer, H. (2004). Control of rRNA synthesis in Escherichia coli: a systems biology approach. Microbiol. Mol. Biol. Rev. 68, 639-668. doi: 10.1128/MMBR.68.4.639-668.2004

Eickhoff, M., Fei, C., Huang, X., and Bassler, B. (2021). LuxT controls specific quorum-sensing-regulated behaviors in Vibrionaceae spp. via repression of qrr1, encoding a small regulatory RNA. PLoS Genet. 17:e1009336. doi: 10.1371/journal.pgen.1009336

Ferreira, R. B., Antunes, L. C., Greenberg, E. P., and McCarter, L. L. (2008). Vibrio parahaemolyticus ScrC modulates cyclic dimeric GMP regulation of gene expression relevant to growth on surfaces. J. Bacteriol. 190, 851-860. doi: $10.1128 /$ JB.01462-07

Ferreira, R. B., Chodur, D. M., Antunes, L. C., Trimble, M. J., and McCarter, L. L. (2012). Output targets and transcriptional regulation by a cyclic dimeric GMP-responsive circuit in the Vibrio parahaemolyticus Scr network. J. Bacteriol. 194, 914-924. doi: 10.1128/JB.05807-11

Finkel, S., and Johnson, R. (1992). The Fis protein: it's not just for DNA inversion anymore. Mol. Microbiol. 6, 3257-3265. doi: 10.1111/j.1365-2958.1992. tb02193.x

Froelich, B., and Daines, D. (2020). In hot water: effects of climate change on vibrio-human interactions. Environ. Microbiol. 22, 4101-4111. doi: $10.1111 / 1462-2920.14967$

Froelich, B., and Noble, R. (2016). Vibrio bacteria in raw oysters: managing risks to human health. Philos. Trans. R. Soc. Lond. Ser. B Biol. Sci. 371:20150209. doi: $10.1098 /$ rstb.2015.0209

Fuqua, W. C., Winans, S. C., and Greenberg, E. P. (1994). Quorum sensing in bacteria: the LuxR-LuxI family of cell density-responsive transcriptional regulators. J. Bacteriol. 176, 269-275. doi: 10.1128/jb.176.2.269-275.1994

Gawade, P., Gunjal, G., Sharma, A., and Ghosh, P. (2020). Reconstruction of transcriptional regulatory networks of Fis and H-NS in Escherichia coli from genome-wide data analysis. Genomics 112, 1264-1272. doi: 10.1016/j. ygeno.2019.07.013

Gibson, D. G. (2011). Enzymatic assembly of overlapping DNA fragments. Methods Enzymol. 498, 349-361. doi: 10.1016/b978-0-12-385120-8.00015-2

Gode-Potratz, C. J., Kustusch, R. J., Breheny, P. J., Weiss, D. S., and McCarter, L. L. (2011). Surface sensing in Vibrio parahaemolyticus triggers a programme of gene expression that promotes colonization and virulence. Mol. Microbiol. 79, 240-263. doi: $10.1111 / j .1365-2958.2010 .07445 . x$

Gode-Potratz, C. J., and McCarter, L. L. (2011). Quorum sensing and silencing in Vibrio parahaemolyticus. J. Bacteriol. 193, 4224-4237. doi: 10.1128/ JB.00432-11

Goldberg, M. D., Johnson, M., Hinton, J. C., and Williams, P. H. (2001). Role of the nucleoid-associated protein Fis in the regulation of virulence properties of enteropathogenic Escherichia coli. Mol. Microbiol. 41, 549-559. doi: 10.1046/j. 1365-2958.2001.02526.x

González-Gil, G., Bringmann, P., and Kahmann, R. (1996). FIS is a regulator of metabolism in Escherichia coli. Mol. Microbiol. 22, 21-29. doi: 10.1111/ j.1365-2958.1996.tb02652.x

Grainger, D. C., Hurd, D., Goldberg, M. D., and Busby, S. J. (2006). Association of nucleoid proteins with coding and non-coding segments of the Escherichia coli genome. Nucleic Acids Res. 34, 4642-4652. doi: 10.1093/nar/gkl542

Gray, K. M., Passador, L., Iglewski, B. H., and Greenberg, E. P. (1994). Interchangeability and specificity of components from the quorum-sensing regulatory systems of Vibrio fischeri and Pseudomonas aeruginosa. J. Bacteriol. 176, 3076-3080. doi: 10.1128/jb.176.10.3076-3080.1994

Gregory, G. J., Morreale, D. P., Carpenter, M. R., Kalburge, S. S., and Boyd, E. F. (2019). Quorum sensing regulators AphA and OpaR control expression of the operon responsible for biosynthesis of the compatible solute ectoine. Appl. Environ. Microbiol. 85, 1543-1519. doi: 10.1128/aem.01543-19

Güvener, Z., and McCarter, L. (2003). Multiple regulators control capsular polysaccharide production in Vibrio parahaemolyticus. J. Bacteriol. 185, 5431-5441. doi: 10.1128/JB.185.18.5431-5441.2003

Haines-Menges, B., Whitaker, W. B., and Boyd, E. F. (2014). Alternative sigma factor RpoE is important for Vibrio parahaemolyticus cell envelope stress response and intestinal colonization. Infect. Immun. 82, 3667-3677. doi: 10.1128/IAI.01854-14

Hancock, S., Stella, S., Cascio, D., and Johnson, R. (2016). DNA sequence determinants controlling affinity, stability and shape of DNA complexes bound by the nucleoid protein Fis. PLoS One 11:e0150189. doi: 10.1371/ journal.pone.0150189

Hengen, P., Lyakhov, I., Stewart, L., and Schneider, T. (2003). Molecular flipflops formed by overlapping Fis sites. Nucleic Acids Res. 31, 6663-6673. doi: $10.1093 /$ nar/gkg877

Ho, S. N., Hunt, H. D., Horton, R. M., Pullen, J. K., and Pease, L. R. (1989). Site-directed mutagenesis by overlap extension using the polymerase chain reaction. Gene 77, 51-59. doi: 10.1016/0378-1119(89)90358-2

Ishihama, A. (2010). Prokaryotic genome regulation: multifactor promoters, multitarget regulators and hierarchic networks. FEMS Microbiol. Rev. 34, 628-645. doi: 10.1111/j.1574-6976.2010.00227.x

Jaques, S., and McCarter, L. (2006). Three new regulators of swarming in Vibrio parahaemolyticus. J. Bacteriol. 188, 2625-2635. doi: 10.1128/ JB.188.7.2625-2635.2006

Jones, C., and Wozniak, D. (2017). Congo red stain identifies matrix overproduction and is an indirect measurement for c-di-GMP in many species of bacteria. Methods Mol. Biol. 1657, 147-156. doi: 10.1007/978-1-4939-7240-1_12

Kahramanoglou, C., Seshasayee, A. S. N., Prieto, A. I., Ibberson, D., Schmidt, S., Zimmermann, J., et al. (2011). Direct and indirect effects of H-NS and Fis on global gene expression control in Escherichia coli. Nucleic Acids Res. 39, 2073-2091. doi: 10.1093/nar/gkq934

Kalburge, S. S., Carpenter, M. R., Rozovsky, S., and Boyd, E. F. (2017). Quorum sensing regulators are required for metabolic fitness in Vibrio parahaemolyticus. Infect. Immun. 85, 930-916. doi: 10.1128/iai.00930-16

Karunakaran, R., Mauchline, T. H., Hosie, A. H., and Poole, P. S. (2005). A family of promoter probe vectors incorporating autofluorescent and chromogenic reporter proteins for studying gene expression in Gram-negative bacteria. Microbiology 151, 3249-3256. doi: 10.1099/mic.0.28311-0

Keane, O. M., and Dorman, C. J. (2003). The gyr genes of Salmonella enterica serovar Typhimurium are repressed by the factor for inversion stimulation, Fis. Mol. Gen. Genomics. 270, 56-65. doi: 10.1007/s00438-003-0896-1 
Kelly, A., Goldberg, M. D., Carroll, R. K., Danino, V., Hinton, J. C. D., and Dorman, C. J. (2004). A global role for Fis in the transcriptional control of metabolism and type III secretion in Salmonella enterica serovar Typhimurium. Microbiology 150, 2037-2053. doi: 10.1099/mic.0.27209-0

Kernell Burke, A., Guthrie, L. T. C., Modise, T., Cormier, G., Jensen, R. V., McCarter, L. L., et al. (2015). OpaR controls a network of downstream transcription factors in Vibrio parahaemolyticus BB22OP. PLoS One 10:e0121863. doi: 10.1371/journal.pone.0121863

Kim, Y. K., and McCarter, L. L. (2007). ScrG, a GGDEF-EAL protein, participates in regulating swarming and sticking in Vibrio parahaemolyticus. J. Bacteriol. 189, 4094-4107. doi: 10.1128/JB.01510-06

Klose, K. E., and Mekalanos, J. J. (1998). Distinct roles of an alternative sigma factor during both free-swimming and colonizing phases of the Vibrio cholerae pathogenic cycle. Mol. Microbiol. 28, 501-520. doi: 10.1046/j.1365-2958.1998.00809.x

Kodama, T., Hiyoshi, H., Okada, R., Matsuda, S., Gotoh, K., and Iida, T. (2015). Regulation of Vibrio parahaemolyticus T3SS2 gene expression and function of T3SS2 effectors that modulate actin cytoskeleton. Cell. Microbiol. 17, 183-190. doi: $10.1111 / \mathrm{cmi} .12408$

Lautier, T., and Nasser, W. (2007). The DNA nucleoid-associated protein Fis co-ordinates the expression of the main virulence genes in the phytopathogenic bacterium Erwinia chrysanthemi. Mol. Microbiol. 66, 1474-1490. doi: 10.1111/j. 1365-2958.2007.06012.x

Lenz, D. H., and Bassler, B. L. (2007). The small nucleoid protein Fis is involved in Vibrio cholerae quorum sensing. Mol. Microbiol. 63, 859-871. doi: 10.1111/j. 1365-2958.2006.05545.x

Liu, J., Li, F., and Rozovsky, S. (2013). The intrinsically disordered membrane protein selenoprotein $S$ is a reductase in vitro. Biochemist 52, 3051-3061. doi: $10.1021 /$ bi 4001358

Lubin, J., Lewis, W., Gilbert, N., Weimer, C., Almagro-Moreno, S., Boyd, E., et al. (2015). Host-like carbohydrates promote bloodstream survival of Vibrio vulnificus in vivo. Infect. Immun. 83, 3126-3136. doi: 10.1128/IAI.00345-15

Lv, M., Chen, Y., Liao, L., Liang, Z., Shi, Z., Tang, Y., et al. (2018). Fis is a global regulator critical for modulation of virulence factor production and pathogenicity of Dickeya zeae. Sci. Rep. 8:341. doi: 10.1038/s41598-017-18578-2

Makino, K., Oshima, K., Kurokawa, K., Yokoyama, K., Uda, T., Tagomori, K., et al. (2003). Genome sequence of Vibrio parahaemolyticus: a pathogenic mechanism distinct from that of $V$. cholerae. Lancet 361, 743-749. doi: 10.1016/S0140-6736(03)12659-1

Mallik, P., Pratt, T. S., Beach, M. B., Bradley, M. D., Undamatla, J., and Osuna, R. (2004). Growth phase-dependent regulation and stringent control of fis are conserved processes in enteric bacteria and involve a single promoter (fis $\mathrm{P}$ ) in Escherichia coli. J. Bacteriol. 186, 122-135. doi: 10.1128/JB.186. $1.122-135.2004$

McCarter, L. L. (1998). OpaR, a homolog of Vibrio harveyi LuxR, controls opacity of Vibrio parahaemolyticus. J. Bacteriol. 180, 3166-3173. doi: 10.1128/ JB.180.12.3166-3173.1998

McCarter, L. (1999). The multiple identities of Vibrio parahaemolyticus. J. Mol. Microbiol. Biotechnol. 1, 51-57.

McCarter, L., Hilmen, M., and Silverman, M. (1988). Flagellar dynamometer controls swarmer cell differentiation of V. parahaemolyticus. Cell 54, 345-351. doi: 10.1016/0092-8674(88)90197-3

McCarter, L. L., and Wright, M. E. (1993). Identification of genes encoding components of the swarmer cell flagellar motor and propeller and a sigma factor controlling differentiation of Vibrio parahaemolyticus. J. Bacteriol. 175, 3361-3371. doi: 10.1128/jb.175.11.3361-3371.1993

McDonald, N., DeMeester, K., Lewis, A., Grimes, C., and Boyd, E. (2018). Structural and functional characterization of a modified legionaminic acid involved in glycosylation of a bacterial lipopolysaccharide. J. Biol. Chem. 293, 19113-19126. doi: 10.1074/jbc.RA118.004966

McLeod, S. M., Aiyar, S. E., Gourse, R. L., and Johnson, R. C. (2002). The C-terminal domains of the RNA polymerase alpha subunits: contact site with Fis and localization during co-activation with CRP at the Escherichia coli proP P2 promoter. J. Mol. Biol. 316, 517-529. doi: 10.1006/jmbi.2001.5391

Miller, M. B., and Bassler, B. L. (2001). Quorum sensing in bacteria. Аnnu. Rev. Microbiol. 55, 165-199. doi: 10.1146/annurev.micro.55.1.165

Miller, M. B., Skorupski, K., Lenz, D. H., Taylor, R. K., and Bassler, B. L. (2002). Parallel quorum sensing systems converge to regulate virulence in Vibrio cholerae. Cell 110, 303-314. doi: 10.1016/S0092-8674(02)00829-2
Miller, K. A., Tomberlin, K. F., and Dziejman, M. (2019). Vibrio variations on a type three theme. Curr. Opin. Microbiol. 47, 66-73. doi: 10.1016/j. mib.2018.12.001

Monteiro, L. M. O., Sanches-Medeiros, A., Westmann, C. A., and Silva-Rocha, R. (2020). Unraveling the complex interplay of Fis and IHF through synthetic promoter engineering. Front. Bioeng. Biotechnol. 8:510. doi: 10.3389/ fbioe.2020.00510

Münch, R., Hiller, K., Barg, H., Heldt, D., Linz, S., Wingender, E., et al. (2003). PRODORIC: prokaryotic database of gene regulation. Nucleic Acids Res. 31, 266-269. doi: 10.1093/nar/gkg037

Nair, G. B., Ramamurthy, T., Bhattacharya, S. K., Dutta, B., Takeda, Y., and Sack, D. A. (2007). Global dissemination of Vibrio parahaemolyticus serotype O3:K6 and its serovariants. Clin. Microbiol. Rev. 20, 39-48. doi: 10.1128/ CMR.00025-06

Nealson, K. H., Platt, T., and Hastings, J. W. (1970). Cellular control of the synthesis and activity of the bacterial luminescent system. J. Bacteriol. 104 313-322. doi: 10.1128/jb.104.1.313-322.1970

O'Boyle, N., and Boyd, A. (2014). Manipulation of intestinal epithelial cell function by the cell contact-dependent type III secretion systems of Vibrio parahaemolyticus. Front. Cell. Infect. Microbiol. 3:114. doi: 10.3389/ fcimb.2013.00114

Osuna, R., Lienau, D., Hughes, K. T., and Johnson, R. C. (1995). Sequence, regulation, and functions of fis in Salmonella typhimurium. J. Bacteriol. 177, 2021-2032. doi: 10.1128/jb.177.8.2021-2032.1995

Ouafa, Z. A., Reverchon, S., Lautier, T., Muskhelishvili, G., and Nasser, W. (2012). The nucleoid-associated proteins H-NS and FIS modulate the DNA supercoiling response of the pel genes, the major virulence factors in the plant pathogen bacterium Dickeya dadantii. Nucleic Acids Res. 40, 4306-4319. doi: $10.1093 / \mathrm{nar} / \mathrm{gks} 014$

Philippe, N., Alcaraz, J. P., Coursange, E., Geiselmann, J., and Schneider, D. (2004). Improvement of pCVD442, a suicide plasmid for gene allele exchange in bacteria. Plasmid 51, 246-255. doi: 10.1016/j.plasmid.2004.02.003

Prigent-Combaret, C., Zghidi-Abouzid, O., Effantin, G., Lejeune, P., Reverchon, S., and Nasser, W. (2012). The nucleoid-associated protein Fis directly modulates the synthesis of cellulose, an essential component of pellicle-biofilms in the phytopathogenic bacterium Dickeya dadantii. Mol. Microbiol. 86, 172-186. doi: $10.1111 / j .1365-2958.2012 .08182 . x$

Prouty, M. G., Correa, N. E., and Klose, K. E. (2001). The novel sigma54- and sigma28-dependent flagellar gene transcription hierarchy of Vibrio cholerae. Mol. Microbiol. 39, 1595-1609. doi: 10.1046/j.1365-2958.2001.02348.x

Rutherford, S. T., van Kessel, J. C., Shao, Y., and Bassler, B. L. (2011). AphA and LuxR/HapR reciprocally control quorum sensing in vibrios. Genes Dev. 25, 397-408. doi: 10.1101/gad.2015011

Schneider, D. A., Ross, W., and Gourse, R. L. (2003). Control of rRNA expression in Escherichia coli. Curr. Opin. Microbiol. 6, 151-156. doi: 10.1016/ S1369-5274(03)00038-9

Schneider, R., Travers, A., Kutateladze, T., and Muskhelishvili, G. (1999). A DNA architectural protein couples cellular physiology and DNA topology in Escherichia coli. Mol. Microbiol. 34, 953-964. doi: 10.1046/j.1365-2958.1999.01656.x

Shao, Y., Feldman-Cohen, L., and Osuna, R. (2008). Functional characterization of the Escherichia coli Fis-DNA binding sequence. J. Mol. Biol. 376, 771-785. doi: 10.1016/j.jmb.2007.11.101

Simpson, C., Petersen, B., Haas, N., Geyman, L., Lee, A., Podicheti, R., et al. (2021). The quorum-sensing systems of Vibrio campbellii DS40M4 and BB120 are genetically and functionally distinct. Environ. Microbiol. 23, 5412-5432. doi: 10.1111/1462-2920.15602

Skoko, D., Yoo, D., Bai, H., Schnurr, B., Yan, J., McLeod, S., et al. (2006). Mechanism of chromosome compaction and looping by the Escherichia coli nucleoid protein Fis. J. Mol. Biol. 364, 777-798. doi: 10.1016/j.jmb.2006.09.043

Steen, J. A., Harrison, P., Seemann, T., Wilkie, I., Harper, M., Adler, B., et al (2010). Fis is essential for capsule production in Pasteurella multocida and regulates expression of other important virulence factors. PLoS Pathog. 6:e1000750. doi: 10.1371/journal.ppat.1000750

Stewart, B. J., and McCarter, L. L. (2003). Lateral flagellar gene system of Vibrio parahaemolyticus. J. Bacteriol. 185, 4508-4518. doi: 10.1128/ JB.185.15.4508-4518.2003

Su, Y. C., and Liu, C. (2007). Vibrio parahaemolyticus: a concern of seafood safety. Food Microbiol. 24, 549-558. doi: 10.1016/j.fm.2007.01.005 
Swift, S., Downie, J., Whitehead, N., Barnard, A., Salmond, G., and Williams, P. (2001). Quorum sensing as a population-density-dependent determinant of bacterial physiology. Adv. Microb. Physiol. 45, 199-270. doi: 10.1016/ s0065-2911(01)45005-3

Trimble, M. J., and McCarter, L. L. (2011). Bis-(3'-5')-cyclic dimeric GMPlinked quorum sensing controls swarming in Vibrio parahaemolyticus. Proc. Natl. Acad. Sci. U. S. A. 108, 18079-18084. doi: 10.1073/pnas.1113790108

Wang, H., Liu, B., Wang, Q., and Wang, L. (2013). Genome-wide analysis of the salmonella Fis regulon and its regulatory mechanism on pathogenicity islands. PLoS One 8:e64688. doi: 10.1371/journal.pone.0064688

Weinstein-Fischer, D., and Altuvia, S. (2007). Differential regulation of Escherichia coli topoisomerase I by Fis. Mol. Microbiol. 63, 1131-1144. doi: 10.1111/j. 1365-2958.2006.05569.x

Whitaker, W. B., Parent, M. A., Boyd, A., Richards, G. P., and Boyd, E. F. (2012). The Vibrio parahaemolyticus ToxRS regulator is required for stress tolerance and colonization in a novel orogastric streptomycin-induced adult murine model. Infect. Immun. 80, 1834-1845. doi: 10.1128/IAI.06284-11

Whitaker, W. B., Richards, G. P., and Boyd, E. F. (2014). Loss of sigma factor RpoN increases intestinal colonization of Vibrio parahaemolyticus in an adult mouse model. Infect. Immun. 82, 544-556. doi: 10.1128/IAI. 01210-13

Yang, H., de Souza Santos, M., Lee, J., Law, H. T., Chimalapati, S., Verdu, E. F., et al. (2019). A novel mouse model of enteric Vibrio parahaemolyticus infection reveals that the type III secretion system 2 effector VopC plays a key role in tissue invasion and gastroenteritis. mBio 10, 2608-2619. doi: 10.1128/mBio.02608-19

Zhang, Y., Qiu, Y., Tan, Y., Guo, Z., Yang, R., and Zhou, D. (2012). Transcriptional regulation of opaR, qrr2-4 and aphA by the master quorum-sensing regulator OpaR in Vibrio parahaemolyticus. PLoS One 7:e34622. doi: 10.1371/journal. pone. 0034622

Conflict of Interest: The authors declare that the research was conducted in the absence of any commercial or financial relationships that could be construed as a potential conflict of interest.

Publisher's Note: All claims expressed in this article are solely those of the authors and do not necessarily represent those of their affiliated organizations, or those of the publisher, the editors and the reviewers. Any product that may be evaluated in this article, or claim that may be made by its manufacturer, is not guaranteed or endorsed by the publisher.

Copyright (c) 2021 Tague, Regmi, Gregory and Boyd. This is an open-access article distributed under the terms of the Creative Commons Attribution License (CC BY). The use, distribution or reproduction in other forums is permitted, provided the original author(s) and the copyright owner(s) are credited and that the original publication in this journal is cited, in accordance with accepted academic practice. No use, distribution or reproduction is permitted which does not comply with these terms. 